\title{
Minimal Selectors and Fault Tolerant Networks*
}

\author{
Omid Amini ${ }^{1,2}$ \\ Frédéric Giroire ${ }^{2,3}$ \\ Florian Huc $^{2}$ \\ Stéphane Pérennes ${ }^{2}$
}

\begin{abstract}
In this paper we study a combinatorial optimization problem arising from on-board networks in satellites. In these kinds of networks the entering signals (inputs) should be routed to amplifiers (outputs). The connections are made via expensive switches with four available links. The paths connecting inputs to outputs should be link-disjoint. More formally, we call a $(p, \lambda, k)$-network an undirected graph with $p+\lambda$ inputs, $p+k$ outputs and internal vertices of degree four. A $(p, \lambda, k)$-network is valid if it is tolerant to a restricted number of faults in the network, i.e., if, for any choice of at most $\lambda$ faulty inputs and $k$ faulty outputs, there exist $p$ edge-disjoint paths from the remaining inputs to the remaining outputs.

Our optimization problem consists in determining $N(p, \lambda, k)$, the minimum number of vertices in a valid $(p, \lambda, k)$-network. We present validity certificates and a quasi-partitioning technique from which we derive lower bounds for $N(p, \lambda, k)$. We also provide constructions, and hence upper bounds, based on expanders. The problem is shown to be sensitive to the order of $\lambda$ and $k$. For instance, when $\lambda$ and $k$ are small compared to $p$, the question reduces to the avoidance of some forbidden local configurations. For larger values of $\lambda$ and $k$, the problem is to find graphs with a good expansion property for small sets. This leads us to introduce a new parameter called $\alpha$-robustness. We use $\alpha$-robustness to generalize our constructions for larger values of $k$ and $\lambda$. In many cases, we provide asymptotically tight bounds for $N(p, \lambda, k)$.
\end{abstract}

Keywords: Fault Tolerant Networks, Switching Networks, Network Design, Concentrators, Routing, Connectivity, Disjoint paths, Expanders.

\section{Introduction}

Motivation. In this paper we study a network-design combinatorial optimization problem. Our problem, originally posed by AlCATEL SPACE Industry, comes from the design of efficient on-board networks in satellites (also called Traveling Wave Tube Amplifiers). The satellites under consideration are used for TV and video transmission (like the Eutelsat or Astra series) as well as for private applications. Signals incoming to a telecommunication satellite through ports have to be routed

\footnotetext{
*This work has been partially supported by European project IST FET AEOLUS.

${ }^{1}$ École Polytechnique, Palaiseau, FRANCE, firstname.lastname@polytechnique.org

${ }^{2}$ Mascotte, joint project -INRIA/CNRS-I3S/UNSA- 2004, route des Lucioles - SophiaAntipolis, FRANCE, firstname.lastname@sophia.inria.fr

${ }^{3}$ This work was done while in the Algorithms project, INRIA Rocquencourt, F-78153 Le Chesnay, France.
} 
through an on-board network to amplifiers. four links. But other constraints appear. Two difficulties appear. On the one hand, the amplifiers may fail during the satellite's life time and can not be repaired. On the other hand, as the satellite is rotating, not all the ports are well oriented and hence available. So more amplifiers and ports are needed than the number of signals which have to be routed. Notice that in this context, on the contrary to classical network design studies, we do not consider link and switch failure. As a matter of fact the links are just big tubes and the switches are very reliable rotating mechanical systems.

One can easily construct a network fulfilling these constraints by using a permutation network or two concentrators of any fixed degree. However, to decrease launch costs, it is crucial to minimize the network physical weight, i.e., to minimize the number of switches. Switches are also expensive to build, so it is worth saving even one. Space industries are interested in designing such networks for specific values of the parameters, however the general theory is of interest by itself. Also, due to the increasing launch capacities and the decreasing switch sizes, larger networks will be used in a near future.

Problem. A network can be seen as a graph with a set of inputs and outputs. The vertices of this graph represent the switches. We define a $(p, \lambda, k)$-network as a network with $p+\lambda$ inputs and $p+k$ outputs. A $(p, \lambda, k)-$ network is said to be valid if it can tolerate up to $\lambda$ faults in inputs and up to $k$ faults in outputs. More formally for any choice of $p$ inputs and $p$ outputs, there exist $p$ edge-disjoint paths linking all the chosen inputs to all the chosen outputs. By symmetry, we may assume in the following that $k \geq \lambda$ and we denote the total number of outputs by $n:=p+k$. Figure 1 gives an example of a $(12,4,4)$ valid network with 20 switches (the inputs are represented with arrows, the outputs with squares and the switches with black vertices).

The problem is to find $N(p, \lambda, k)$, the minimum number of switches in a valid $(p, \lambda, k)$-network, and to give constructions of such networks. Note that finding minimal networks is a challenging problem and even testing the validity of a given network is hard (see related work). We study the case where the switches of the network have degree four (although the theory can be generalized to any degree) which is of primary interest for the applications.

Related Work. This study of a new kind of networks should be considered in the spirit of the well studied theory of superconcentrators. A $(p+k, p)$-concentrator [31] is a directed acyclic graph $G=(V, E)$ with $p+k$ designated input vertices and $p$ designated output vertices $(k \geq 0)$, such that for each subset of $p$ input vertices, there exist $p$ edge-disjoint (or, depending on the context, vertex-disjoint) paths from the input vertices to the output vertices. A selector, first introduced in [9], is a $(p, 0, k)$-network $(\lambda=0)$. A general theory of selectors can be found in [12], where several results are obtained for small values of $k$. A selector can be seen as an undirected version of a $(p+k, p)$-concentrator by changing the role of inputs and outputs (and by adding the degree condition). An $n$-superconcentrator [28] is a directed acyclic graph $G=(V, E)$ with $n$ designated input vertices and $n$ designated output vertices such that, for any set $S$ of $p \leq n$ inputs and any set $T$ of $p$ outputs, there exist $p$ edge-disjoint (or, vertex-disjoint depending on the context) paths connecting $S$ to $T$. There is a vast theory of superconcentrators (see the seminal work of Pippenger [28] for an introduction, [2, 3, 5, 30] for constructions, and [14] for complexity issues). 
In this way, $(p, \lambda, k)$-networks generalize the concept of selectors as superconcentrators generalize concentrators. It is also clear that taking a superconcentrator (resp. $(p+k, p)$-concentrator) and forgetting the orientations provides a valid $(p, \lambda, k)$-network (resp. selector) for every value of $k=\lambda$ (resp. any $k$ and $\lambda=0$ ), once the degree condition is respected. So one can try to use superconcentrators to construct efficient on-board satellite networks, but, not surprisingly, this does not give minimal networks in general. The major difference between the superconcentrators and the general valid networks, beside the degree condition, is that in a valid network the number of inputs and outputs that may become out of service is bounded (in our case, given a failure probability, no more than $k$ failures will occur during the satellite lifetime). Finding a minimum network (a superconcentrator of low density or having a minimum number of vertices) is a challenging problem and is an active area of research. From the algorithmic point of view, one can show that the problem of testing if a graph is a $(p+k, p)$-concentrator or a superconcentrator is coNP-complete. In fact, this is coNP-complete even when restricted to the important special case of graphs of size linear in the number of inputs (see [14]). By the same arguments one can prove that the problem of deciding if a given $(p, \lambda, k)$-network is valid or not is coNP-complete.

In [12] the authors consider a variant of selectors where some signals have priority and should be sent to amplifiers offering the best quality of service. In [8] the authors study the case where all the amplifiers are different and where a given input has to be sent to a dedicated output. This problem is related to permutation networks.

In $[11,21]$, the authors present exact values of $N(p, \lambda, k)$ for small values of $k$ and $\lambda$ and arbitrary values of $p$. For instance $N(p, 2,1)=N(p, 1,2)=N(p, 2,2)=p+2$ and also for $k \in\{3,4\}$ and $0<\lambda \leq 4, N(p, \lambda, k)=\left\lceil\frac{5 n}{4}\right\rceil$.

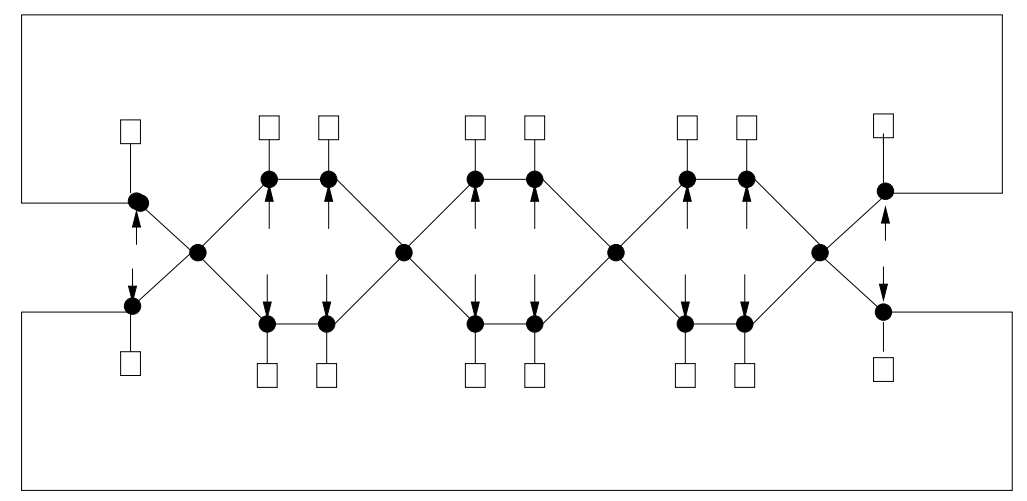

Figure 1: A valid $(12,4,4)$-network with the minimum number of switches.

Results. In this paper we are primarily interested in large networks, i.e., where $n=p+k$ tends to infinity and also $k$ is large enough. We present several upper and lower bounds for the minimum number of switches in a valid $(p, \lambda, k)$-network. In many cases these bounds are asymptotically tight. Since forgetting the orientation of superconcentrators provides valid $(p, \lambda, k)$-networks, these results also provide lower bounds on the size of a superconcentrator. To obtain the lower bounds we present a new technique, quasi-partitioning. We give here the very first applica- 
tions of this lemma, but we think it may have several applications in other related problems (for example bisection-width, see [24]).

To obtain upper bounds we propose several constructions. The constructions of optimal valid networks will heavily rely on expanders (see Section 4.1). Using these, we are able to construct, for a special class of networks called simplified networks, networks with $2 n$ switches as soon as $n$ is large enough and $k \leq c_{1} \log n$ for some constant $c_{1}$ (Section 4.3). In Section 6 we also give a lower bound of order $2 n(1-\epsilon(k))$ where $\epsilon(k)$ tends to zero when $k$ tends to infinity (but we do not need $k \leq c_{1} \log n$ ). Thus for simplified networks the problem is asymptotically solved for

$k \leq c_{1} \log n$. For general networks, using bipartite expanders, we obtain an upper bound of $n+\frac{3}{4} n$ when $k \leq c_{2} \log n$ for some constant $c_{2}$. The lower bound we obtain is $\left(n+\frac{2}{3} n\right)(1-\epsilon(\lambda, k))$, and we conjecture that $n+\frac{3}{4} n$ should be the right value. We also give a construction of selectors (case $\lambda=0$ ) of size $n+\frac{n}{2}$, in which case we get also a tight lower bound.

To extend the results to larger values of $k$, we define a local expansion property of graphs that we call $\alpha$-robustness (see $[4,16]$ for some related notions). Intuitively, the $\alpha$-robustness of a graph $G$ is a local version of the expansion factor. It is the maximum integer $r_{\alpha}$ such that for small subsets the expansion factor is $\alpha$ but for larger subsets, the minimum number of outgoing edges is required to be at least $r_{\alpha}$. In our constructions, we only need our graph to be an expander for small subsets, but for larger subsets all we need is a minimum constant edge-connectivity to the rest of the graph. This notion is also interesting in its own right, and contains several expansion-invariants such as bisection-width and Cheeger's constant as special cases (see [18]). As random graphs are very good expanders, we study the $\alpha$-robustness of random graphs. In this way we generalize Bollobàs' result on the expansion factor of random 4-regular graphs (see [15]) to obtain related results for $\alpha$-robustness of random 4-regular graphs. As far as we know it is the first time in the literature that this new concept of local expanders is defined and investigated. Using this we present a construction of valid $(p, \lambda, k)$-networks with $3 n$ switches for $\lambda \leq k \leq \frac{n}{7}$.

\section{Preliminaries}

In this section, we define more formally the design problem and introduce notation used throughout the paper. We state a cut criterion (Proposition 1): this criterion is fundamental because it characterizes the validity of $(p, \lambda, k)$-networks. It is extensively used to prove that networks are valid - in particular for the networks of Section 4. In Section 6 we also use the cut criterion to detect forbidden patterns leading to lower bounds for the number of switches of valid networks. Proofs of lower and upper bounds are simplified by the use of the last notion introduced here, the associated graph of a network (see Section 4.2 and Section 6.1).

Notation. Given a function $f$, we define $f(A):=\sum_{a \in A} f(a)$ for any finite set $A$. For a subset $W$ of vertices of a graph $G=(V, E)$, let us denote by $\Delta(W)$ the set of edges connecting $W$ and $\bar{W}=V \backslash W$ and by $\Gamma(W)$ the set of vertices of $\bar{W}$ adjacent to a vertex of $W$. If a set is denoted by an upper case letter, the corresponding lower case letter denotes its cardinality (for example $\delta(W)=|\Delta(W)|$ ). 
$(p, \lambda, k)$-Networks and Valid $(p, \lambda, k)$-Networks. A $(p, \lambda, k)$-network is a triple $\mathcal{N}=\{(V, E), i, o\}$ where $(V, E)$ is a graph and $i, o$ are non-negative integer functions defined on $V$ called input and output functions, such that for any $v \in V$, $i(v)+o(v)+\operatorname{deg}(v)=4$. The total number of inputs is $i(V)=\Sigma_{v \in V} i(v)=p+\lambda$, and the total number of outputs is $o(V)=\Sigma_{v \in V} o(v)=p+k$. We can see a network as a graph where all vertices but the leaves have degree four, and in which inputs and outputs are leaves. A non-faulty output function is a function $o^{\prime}$ defined on $V$ such that $o^{\prime}(v) \leq o(v)$ for any $v \in V$ and $o^{\prime}(V)=p$. A used input function is a function $i^{\prime}$ defined on $V$ such that $i^{\prime}(v) \leq i(v)$ for any $v \in V$ and $i^{\prime}(V)=p$. A $(p, \lambda, k)$-network is said valid if for any non-faulty output function $o^{\prime}$ and any used input function $i^{\prime}$, there are $p$ edge-disjoint paths in $G$ such that each vertex $v \in V$ is the initial vertex of $i^{\prime}(v)$ paths and the terminal vertex of $o^{\prime}(v)$ paths.

Design Problem and Simplified Design Problem. Let $N(p, \lambda, k)$ denote the minimum number of switches of a valid $(p, \lambda, k)$-network. The Design Problem consists of determining $N(p, \lambda, k)$ and constructing a minimum $(p, \lambda, k)$-network, or at least a valid $(p, \lambda, k)$-network with a number of switches close to the optimal value. We introduce a variation of the problem, the Simplified Design Problem, which is to find minimum networks having $p+\lambda$ switches with one input and one output and $k-\lambda$ switches with only one output. Networks of this kind are especially good for practical applications, as they simplify the routing process, minimize path lengths and lower interferences between signals.

Excess, Validity and Cut Criterion. To verify if a network is valid, instead of solving a flow/supply problem for each possible configuration of output failures and of used inputs, it is sufficient to look at an invariant measure of subsets of the network, the excess, as expressed in the following proposition.

Proposition 1 (Cut Criterion) $A(p, \lambda, k)$-network is valid if and only if, for any subset of vertices $W \subset V$ the excess of $W$, defined by

$$
\varepsilon(W):=\delta(W)+o(W)-\min (k, o(W))-\min (i(W), p),
$$

satisfies $\varepsilon(W) \geq 0$.

The intuition is that the signals arriving in $W$ (in number at most $\min (i(W), p)$ ) should be routed either to the valid outputs of $W$ (in number at least $o(W)-$ $\min (k, o(W)))$ or to the links going outside (in number $\delta(W)$ ). The omitted formal proof reduces to a supply/demand flow problem. Note that, for the cut criterion, it is sufficient to consider only connected subsets $W$ with connected complement $\bar{W}$ (this comes from the submodularity of $\varepsilon$ ).

Associated Graph. Vertices $x \in V$ of degree two with $i(x)=o(x)=1$ play an important role. We call them doublons. A switch that is not a doublon is called an $R$-switch. Remark that for $k \geq 3$ no paths has three or more consecutive doublons. Indeed if we consider the set $W$ consisting of these doublons we have $\delta(W)=2$ and $o(W)=i(W) \geq 3$. The cut criterion would give a contradiction.

Let $\mathcal{N}$ be a $(p, \lambda, k)$-network. We build a graph $\mathcal{R}$ associated with $\mathcal{N}$. Its vertices are the $R$-switches of $\mathcal{N}$. Consequently the edges of $\mathcal{R}$ are of three kinds, respectively $E_{0}, E_{1}$ and $E_{2}$ : the edges of $\mathcal{N}$ between two $R$-switches, the edges corresponding in $\mathcal{N}$ to a path of length two with a doublon in the middle and those corresponding to a path of length three with two doublons in the middle. 


\section{Summary of Main Results}

The aim of this short section is to provide an overview of the results obtained in the upcoming sections.

The design problem is treated in Sections 4 and 5 . For relatively small values of $k$ and $\lambda$, we provide constructions of valid networks for the general design problem, the design problem when $\lambda=0$ (selectors) and the simplified design problem. More precisely, we prove the following theorems:

Theorem 2 of Section 4 (General Design Problem). Let $n=p+k, k \leq \frac{1}{15} \log n$, for $n$ large enough, we have: $N(p, \lambda, k) \leq n+\frac{3}{4} n$

Theorem 3 of Section 4 (Selectors, $\lambda=0$ ). Let $n=p+k, k \leq \frac{1}{48} \log _{2} n$, for $n$ large enough, we have:

$$
N(p, 0, k) \leq n+\frac{n}{2}
$$

Theorem 1 of Section 4 (Simplified Design Problem). Let $n=p+k, k \leq \frac{1}{6} \log n$, for $n$ large enough, we have: $N(p, \lambda, k) \leq 2 n$.

In Section 5, we provide a general framework to extend the above mentioned results to larger values of $\lambda$ and $k$. We define a new parameter, which we call robustness, which captures the local expansion property of a given graph. We study the robustness of random regular graphs and use these graphs for the design problem. In particular, we prove

Theorem 4 of Section 5. Random 4-regular graphs have 1-robustness at least $\frac{n}{14}$ with high probability, i.e., with probability $1-\mathcal{O}\left(\frac{\log n}{n}\right)$.

Corollary 1 of Section 5 . For $k \leq \frac{n}{7}$, there exist $(p, \lambda, k)$-valid networks of size $3 n$ (remember that $n=p+k$ ).

The lower bounds, confirming the tightness of the above results in many cases, are provided in Section 6. We first prove a technical quasi-partition lemma:

Lemma 4 of Section 6. Let $q$ be a positive integer and let $G$ be a connected graph of order at least $\frac{q}{3}$. Then $G$ admits a $q$-quasi-partition (for the definition see Section 6).

Using this lemma, we prove the following general theorem:

Theorem 5. In a valid network $\mathcal{N}$ of size $N$, with $k \leq \frac{n}{2}$, we have

$$
\begin{aligned}
N & \geq\left(\frac{3}{2} n-(k-\lambda)-4-\frac{12}{\sqrt{k}}\right)\left(1-\frac{9}{2 \sqrt{k}}+O\left(\frac{1}{k}\right)\right) \\
& +\frac{d}{2}\left(1+\frac{9}{2 \sqrt{k}}+O\left(\frac{1}{k}\right)\right) .
\end{aligned}
$$

where $n=p+k$ and $d$ is the number of doublons, i.e., switches with exactly one input and one output.

Corollaries of this theorem are 
Theorem 8 of Section 6 (General case). When $\lambda \rightarrow \infty$ and $k \rightarrow \infty$, any valid network has size at least $n+\frac{2}{3} n+O\left(\frac{n}{\sqrt{\lambda}}\right)$. In particular, $N(p, \lambda, k) \geq n+\frac{2}{3} n+O\left(\frac{n}{\sqrt{\lambda}}\right)$.

Theorem 6 of Section 6 (Selectors). In a valid selector $\mathcal{N}$ of size $N$, when $k \rightarrow \infty$ with $k \leq \frac{n}{2}$, we have

$$
N \geq n+\frac{n}{2}+\mathcal{O}\left(\frac{n}{\sqrt{k}}\right)
$$

In other words

$$
N(p, 0, k) \geq n+\frac{n}{2}+O\left(\frac{n}{\sqrt{k}}\right) .
$$

Theorem 7 of Section 6 (Simplified case). In the simplified case, when $k \rightarrow$ $\infty$ with $k \leq \frac{n}{2}$, every valid network has size at least $2 n+O\left(\frac{n}{\sqrt{k}}\right)$, i.e., we have $N(p, \lambda, k) \geq 2 n+O\left(\frac{n}{\sqrt{k}}\right)$ in the simplified case.

\section{Upper Bounds: The Design Problem}

In this section, we give constructions that rely heavily on expanders. We present here three constructions with $2 n, n+\frac{3}{4} n$ and $n+\frac{n}{2}$ switches respectively for the simplified design problem, the design problem (any $\lambda$ ) and for the design problem when $\lambda=0$. All these constructions are valid for $k \leq c \cdot \log n$ (where $c$ is a constant depending only on the expansion factor of 3 and 4 -regular graphs).

\subsection{Expanders}

An expander (see [26, 27] for surveys) is a highly connected sparse graph. They are used in various fields of computer science and mathematics. For example they are valuable in constructions of error-correcting codes with efficient encoding and decoding algorithms, derandomization of random algorithms, and embeddings of finite metric spaces. But they also have applications in areas directly related to the subject of this paper as in the design of explicit superefficient networks and the explicit construction of graphs with large girth (length of the smallest cycle). We present here known results about expanders that are used in proofs of Sections 4.3, 4.4 and 4.5. The formal definition of an expander is as follows: an $(n, r, c)-E$ expander is a finite $r$-regular graph $G=(V, E)$ with $n$ vertices such that for any set $A$ of vertices of $G$ with $|A| \leq|V| / 2$ we have $\delta(A) \geq c|A|$. Well known examples of expanders are Ramanujan graphs (for more on Ramanujan graphs see [25] and the book [19]). Explicit constructions of Ramanujan graphs are known for $r$ of the form $r=q+1$, with $q$ a prime power (in particular for $r=3$ and $r=4$, which are of special interest in our case). More precisely there exist explicit constructions of an infinite family $G_{i}=\left(V_{i}, E_{i}\right)$ of Ramanujan graphs such that $\left|V_{i}\right| \underset{i \rightarrow \infty}{\rightarrow} \infty$ with an expansion factor $c \geq 1-\frac{4(r-1)}{r^{2}}$. It gives $c \geq \frac{1}{4}$ for 4 -regular graphs and $c \geq \frac{1}{9}$ for 3 -regular graphs. The girth of the graphs of this family satisfies: $g\left(G_{i}\right) \geq \frac{2}{3} \log _{q}\left|V_{i}\right|$. There also exists a family $H_{i}=\left(W_{i}, F_{i}\right)$ of explicit bipartite Ramanujan graphs of girth $g\left(H_{i}\right) \geq \frac{4}{3} \log _{q}\left|W_{i}\right|$. Probabilistic arguments show the existence of graphs, for any large order of networks (and not only for the specific values of both families), with the same properties of girth and even better expansion factor: for 4-regular graphs, expanders with $c \geq \frac{11}{25}$ exist (see [15]).

We also need a vertex-expansion version of expanders: 
Definition 1 ( $V$-Expander) An $(n, r, d)$-V-expander is a finite $r$-regular graph $G=$ $(V, E)$ with $|V|=n\left(\left|V_{1}\right|=\left|V_{2}\right|=n\right.$ in case of a bipartite graph and $\left.V=V_{1} \cup V_{2}\right)$ such that for any subset $A$ of vertices $\left(A \subset V_{1}\right.$ when $G$ is bipartite), the set of neighbors of $A, \Gamma(A)=\{v \in V \mid(v, u) \in E$ for some $u \in V\}$ satisfies

$$
|\Gamma(A)| \geq|A|+d(1-|A| / n)|A| .
$$

\subsection{Cut Criterion for the Associated Graph}

To simplify the proofs of validity of Sections 4.3, 4.4 and 4.5 , it is sometimes better to work directly on the associated graph $\mathcal{R}$ of the $(p, \lambda, k)$-network $\mathcal{N}=$ $((V, E), i, o)$. More precisely, it means that, when applying the cut criterion on $\mathcal{N}$, it would be sufficient to consider only subsets of $\mathcal{R}$.

We introduce another notion of excess, $\varepsilon^{\prime}$, defined for all $W \subset V$ as $\varepsilon^{\prime}(W):=$ $\delta(W)+o(W)-\min (k, o(W))-i(W)$. Note that $\varepsilon^{\prime}(W) \leq \varepsilon(W)$. Hence, if, for all $W \subset V, \varepsilon^{\prime}(W) \geq 0$, the network is valid, but we no more have an equivalence. The good property of $\varepsilon^{\prime}$ is that, if a switch $x$ is a doublon, $\varepsilon^{\prime}(W \cup\{x\}) \leq \varepsilon^{\prime}(W)$. So it is enough to verify the cut criterion for subsets $W$ of $\mathcal{N}$ consisting of a set of $R$-switches plus all the doublons on the edges of type $E_{1}$ and $E_{2}$ incident to the $R$-switches.

\subsection{Simplified Design Problem - Upper Bound $2 n$}

In this subsection, we use the existence of expanders presented in Section 4.1 to construct valid $(p, \lambda, k)$-networks with $2 n=2(p+k)$ switches for large $n$ and $k \leq c_{1} \log n$ ( $c_{1}$ depending on the expansion factor of 4 -regular expanders, $c_{1}=\frac{1}{6}$ when using explicit Ramanujan graphs). Furthermore we will show in Section 6 a lower bound of the same order for the simplified design problem.

Theorem 1 Let $n=p+k, k \leq \frac{1}{6} \log n$, for $n$ large enough, we have: $N(p, \lambda, k) \leq$ $2 n$.

Proof The results on expanders presented in Section 4.1 state the existence of an $\left(n, 4, c=\frac{1}{4}\right)$-E-expander, $G=(V, E)$, of girth $g, g \geq \frac{2}{3} \log n$. Let $k \leq c \cdot g$ and $p=n-k$. We claim that in a 4 -regular graph there exists a family of vertexdisjoint cycles covering all vertices of $G$. For now, suppose this is true and call this disjoint union of cycles $F$. We add $n$ new vertices, one on each edge of $F$ by subdividing each edge of $F$ into two edges. On each new vertex, we add an output and input creating a doublon. We now have a $(p, k, k)$-network $\mathcal{N}$ with $2 n$ switches.

Let us prove that the constructed network is valid. We use the cut criterion on $\mathcal{R}$, which, in that case, is exactly $G$. Notice first that the network is symmetric in inputs and outputs and that for any subset $W \subset V$ of vertices, $i(W)=o(W)$. Hence we have

$$
\varepsilon^{\prime}(W)=\delta(W)-\min (k, o(W)) .
$$

Furthermore, note that when a network is symmetric, it is sufficient to verify the cut criterion only for subsets $W$ with $|W| \leq|V| / 2$. 
- If $|W| \geq \frac{k}{c}$ then, by the expansion property, there are at least $k$ edges between $W$ and $\bar{W}$ and so $\varepsilon^{\prime}(W) \geq 0$.

- Otherwise, if $|W|<\frac{k}{c} \leq g$, we have $|W|<g$ and thus $W$ is acyclic. There are at most $|W|-1$ edges inside $W$ and, as $G$ is 4-regular, we have $\delta(W) \geq$ $2|W|+2$. Let $e_{F}(W)$ be the number of edges of $F$ incident to a vertex of $W$; by construction $o(W)=e_{F}(W)$. As the cycles of $F$ are disjoint, $e_{F}(W) \leq 2|W|$. Hence $\delta(W) \geq e_{F}(W)=o(W)$, that is $\varepsilon^{\prime}(W) \geq 0$.

The $(p, k, k)$-network is valid. We can derive $(p, \lambda, k)$-networks for any $\lambda \leq k$ by deleting $k-\lambda$ inputs.

The only remaining point now is to prove the claim. We prove it for any $2 r$ regular graph. Let $G=(V, E)$ be a $2 r$-regular graph. Since $G$ is Eulerian, we can give to $G$ an orientation, such that each vertex has in-degree and out-degree $r$. Let $D=(V, A)$ be the corresponding digraph. Now consider the following bipartite graph: $H=\left(V \times\{1\} \cup V \times\{2\}, E^{\prime}\right)$ where $E^{\prime}=\{((x, 1),(y, 2)) \mid(x, y) \in A\}$. It is clear that $H$ is a bipartite $r$-regular graph, and so it admits a perfect matching. Let $F$ be the edges of $G$ given by this perfect matching. It is easy to check that $F$ is a 2-regular graph covering all the vertices, i.e., $F$ is a disjoint union of cycles covering all the vertices.

\subsection{General Design Problem - Upper Bound $n+\frac{3}{4} n$}

In this subsection, we construct general $N(p, \lambda, k)$-networks for large $n, k \leq c_{2} \log n$ (where $c_{2}$ depends on the expansion factor of 3 -regular expanders). Theorem 2 gives an $n+\frac{3}{4} n$ upper bound for such networks. Constructions are based on 3 regular bipartite expanders.

Definition 2 Two edges are at distance at least $d$ if any path that contains both of them is of length at least $d+2$. A vertex is at distance at least $d$ of an edge if any path that contains both of them is of length at least $d+1$.

Lemma 1 Let $G$ be a 3-regular bipartite graph $G=\left(V_{1} \cup V_{2}, E\right)$ of large girth $\left(g=\Theta\left(\log \left|V_{1}\right|\right)\right)$ which is a $\left(2\left|V_{1}\right|, 3, c\right)$-E-expander and suppose $2 k \leq c g$. Let $\mathcal{F}$ be a set of selected edges, such that any two edges of $\mathcal{F}$ are at distance at least 3 . The network $\mathcal{N}$ obtained from $G$ by adding a doublon on each edge of $\mathcal{F}$, an input on each vertex of $V_{1}$ and an output on each vertex of $V_{2}$ is a valid network.

Proof

We use the cut criterion on the associated graph following Section 4.2. As the construction is symmetric in inputs and outputs, it is sufficient to consider connected subsets $W \in V$ with $|W| \leq\left\lceil\frac{|V|}{2}\right\rceil$. The cut criterion is implied by

$$
\varepsilon^{\prime}(W)=\delta(W)+o(W)-\min (o(W), k)-i(W) \geq 0 .
$$

We now distinguish two cases for $W$.

- case 1: $|W| \leq \frac{2 k}{c} \leq g$.

As $o(W)-\min (o(W), k) \geq 0$, we have

$$
\varepsilon^{\prime}(W) \geq \delta(W)-i(W) .
$$


Hence it is sufficient to prove $\delta(W) \geq i(W)$. As $|W| \leq g$, there are no cycles inside $W$ and therefore there are $|W|-1$ edges inside; $G$ is 3 -regular, so $\delta(W)=|W|+2$. Furthermore, $i(W)=\left|V_{1} \cap W\right|+d$, so we have to prove that $\left|V_{2} \cap W\right|+2 \geq d$.

Consider a doublon $D$ incident to $W$ and its associated edge $e(D)=\left\{v_{1}(D), v_{2}(D)\right\}$ with $v_{1}(D) \in V_{1}$ and $v_{2}(D) \in V_{2}$. If $v_{2}(D) \in W$, associate $D$ with $v_{2}(D)$. If $v_{2}(D) \notin W$, then $v_{1}(D) \in W$. Associate to $D$ a neighboor of $v_{1}(D) \in W$. As the distance of two edges of $\mathcal{F}$ is at least 3 , different doublons have different associate vertices in $V_{2} \cap W$. So $\left|V_{2} \cap W\right| \geq d$.

- case $2:|W| \geq \frac{2 k}{c}$. By definition of $\varepsilon^{\prime}$ we have

$$
\varepsilon^{\prime} \geq \delta(W)+o(W)-k-i(W) .
$$

- If $i(W)-o(W) \leq k$, by the expansion property, we have $\delta(W) \geq 2 k \geq$ $i(W)-o(W)+k$.

- If $i(W)-o(W) \geq k$, since the graph is bipartite, there are at least $3(i(W)-o(W))$ outgoing edges. So $\varepsilon^{\prime}(W) \geq 2(i(W)-o(W))-k \geq k>0$.

Theorem 2 (Construction) Let $n=p+k, k \leq \frac{1}{15} \log n$, for $n$ large enough, we have: $N(p, \lambda, k) \leq n+\frac{3}{4} n$.

Proof Take $H=(A, B, E)$, a bipartite $\left(2|A|, 4, c^{\prime}\right)$-E-expander with girth $g=$ $\frac{4}{3} \log n$. Let $k, k \leq c^{\prime} \cdot g \leq \frac{4}{3} \log n$ (for existence see Section 4.1). Take a complete matching $F$ in the bipartite complement $\bar{H}=(A, B, \bar{E})$ of $H$, that is, every edge $\{u, v\} \in F$, has $u$ and $v$ in different parts of $H$, i.e. $u \in A$ and $v \in B$ OR $u \in B$ and $v \in A$, and $u, v$ are not adjacent in $H$. For each edge $e=\{a, b\}$ of $F, a \in A$ and $b \in B$, replace $a$ and $b$ by three vertices $a_{1}, a_{2}, a_{3}$ and $b_{1}, b_{2}, b_{3}$, add edges $\left\{a_{1}, b_{1}\right\}$, $\left\{a_{1}, b_{2}\right\},\left\{a_{1}, b_{3}\right\},\left\{a_{2}, b_{1}\right\}$ and $\left\{a_{3}, b_{1}\right\}$. Finally join $a_{2}$ (resp $\left.b_{2}\right)$ to two neighbors of $a(\operatorname{resp} b)$ and $a_{3}$ (resp $\left.b_{3}\right)$ to the two other neighbors. See Figure 2. We obtain a 3-regular bipartite graph $G$, which, one can verify, is a $\left(6|A|, 3, \frac{c}{5}\right)-E$-expander. Note that by construction the edges of type $\left\{a_{1}, b_{1}\right\}$, with $\{a, b\} \in F$, form a selected set $\mathcal{F}$ of edges, that are pairwise at distance 3. We can apply Lemma 1 to $G$ with $\mathcal{F}$ as selected set. All together we have $6|A|+|\mathcal{F}|=7|A|$ switches, $\left|V_{1}\right|+|\mathcal{F}|=4|A|$ inputs, $\left|V_{2}\right|+|\mathcal{F}|=4|A|$ outputs. So, with $n=p+k, N(p, k, k)=7|A|=\frac{7}{4} n$. We can now derive $(p, \lambda, k)$-networks for any $\lambda \leq k$ by deleting $k-\lambda$ inputs.

\subsection{Design Problem: $\lambda=0$ - Upper Bound $n+\frac{n}{2}$}

In this subsection, we study selectors (the case $\lambda=0$ ). In Theorem 3 we construct valid $(p, 0, k)$-networks with $n+\frac{n}{2}$ switches for $n$ large and $k \leq c_{3} \log n$ ( $c_{3}$ depends on the vertex-expansion of 3-regular expanders). Such networks are built from bipartite 3 -regular vertex-expanders.

Theorem 3 Let $n=p+k, k \leq \frac{1}{48} \log _{2} n$. For $n$ large enough, we have:

$$
N(p, 0, k) \leq n+\frac{n}{2}
$$




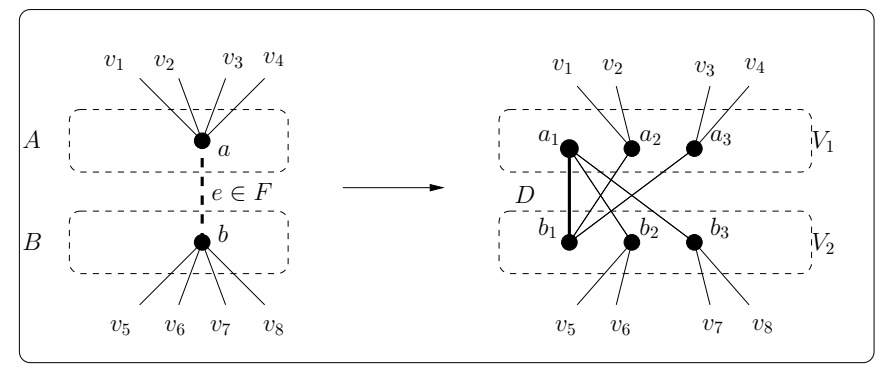

Figure 2: Replacement of a selected couple of a bipartite graph $(A, B, E)$.

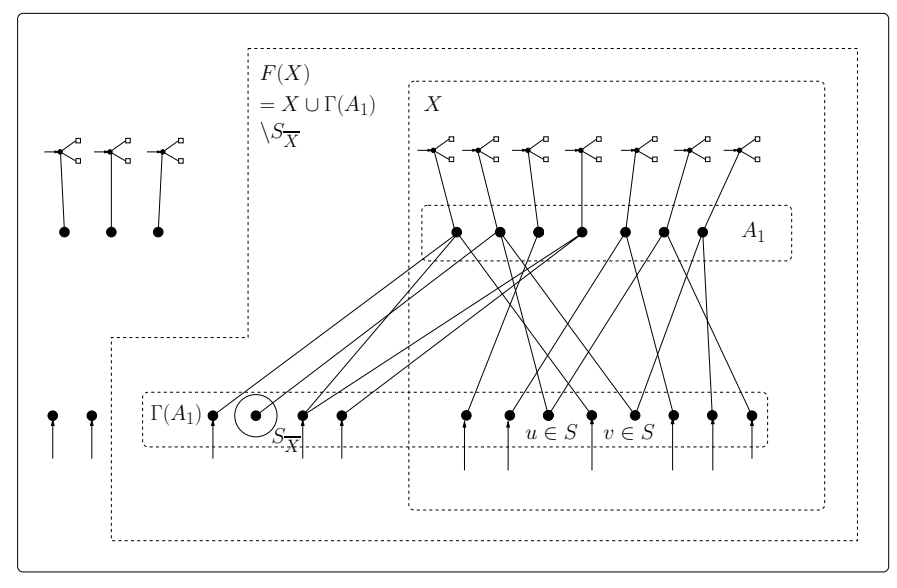

Figure 3: Sketch of proof of Theorem 3.

Proof Let us take $G=\left(V_{1} \cap V_{2}, E\right)$ a bipartite $\left(n, 3, d=\frac{1}{12}\right)-V$-expander of large girth $g \geq \frac{4}{3} \log n$ (for existence see Section 4.1). Let $\alpha=\frac{4}{d}=48$ and $k$ such that $k \leq d \cdot \frac{g}{2} \leq \frac{g}{12}$ and $k \cdot\left(2^{\alpha k}+1\right) \leq n$.

To each vertex of $V_{1}$, we connect a vertex with one input and two outputs. Such a switch is said to be of type $T$. To each vertex of $V_{2}$, we connect an input. We choose a subset $S$ of $k$ vertices in $V_{2}$ such that the distance between any two of them is at least $\alpha \cdot k$ (see Definition 2). By the choice of $k$ we can choose the vertices in $S$ one by one after removing all the vertices at distance less than $\alpha k$ of already chosen vertices (at each step we remove at most $2^{\alpha k}+1$ new vertices). We remove the inputs of all vertices of $S$ obtaining a $(p=n-k, 0, k)$-network that we call $\mathcal{N}=(V, E, i, o)$.

We now prove that this network is valid. Let $X$ be a connected subset of $V$. Let $\bar{X}=V_{1} \cup V_{2} \backslash X, A_{1}=X \cap V_{1}, S_{\bar{X}}=S \cap \Gamma\left(A_{1}\right) \cap \bar{X}$. We define $Z(X)=X \cup \Gamma\left(A_{1}\right) \backslash S_{\bar{X}}$ (Figure 3).

$Z=Z(X)$ is connected. We also have $\varepsilon(Z) \leq \varepsilon(X)$, making it sufficient to verify the cut criterion for $Z$ (same principles as in Section 4.2). Let $A_{2}=Z \cap V_{2}$.

We distinguish four cases for $\left|A_{1}\right|$ :

- $\left|A_{1}\right| \leq \frac{k}{2}$ :

as $o(Z)=2\left|A_{1}\right| \leq k$ and $\varepsilon(Z) \geq \delta(Z)-i(Z)$, we have $\delta(Z) \geq 3\left|A_{2}\right|-3\left|A_{1}\right|$ and $i(Z) \leq\left|A_{2}\right|+\left|A_{1}\right|$. Furthermore there is no cycle in $Z$ so $\left|A_{2}\right| \geq 2\left|A_{1}\right|$. 
Hence $\varepsilon(Z) \geq 0$.

- $\frac{k}{2} \leq\left|A_{1}\right| \leq \frac{k}{d} \leq \frac{g}{2}$ :

we have $\varepsilon=\delta(Z)+o-i-k$ and $i \leq\left|A_{1}\right|+\left|A_{2}\right|$. So $\varepsilon(Z) \geq \delta(Z)-\left|A_{2}\right|+\left|A_{1}\right|-k$. As $Z$ contains no cycle $\delta(Z) \geq 3\left|A_{2}\right|-3\left|A_{1}\right|$ so $\varepsilon \geq 2\left|A_{2}\right|-2\left|A_{1}\right|-k \geq$ $2\left|A_{1}\right|-k \geq 0$. We use again that $\left|A_{2}\right| \geq 2\left|A_{1}\right|$.

- $\frac{k}{d} \leq\left|A_{1}\right| \leq n-\frac{k}{d}$ :

in this case, $\varepsilon \geq \delta(Z)-\left|A_{2}\right|+\left|A_{1}\right|-k$ and $\delta(Z) \geq 3\left(\left|A_{2}\right|-\left|A_{1}\right|\right)=\left|A_{2}\right|-\left|A_{1}\right|+$ $2\left(\left|A_{2}\right|-\left|A_{1}\right|\right)$, so we have $\varepsilon \geq 2\left(\left|A_{2}\right|-\left|A_{1}\right|\right)-k$. Furthermore $\left|A_{2}\right|-\left|A_{1}\right| \geq$ $\Gamma_{G}\left(A_{1}\right)-k$, because at most $k$ vertices of $\Gamma_{G}\left(A_{1}\right)$ are in $S \backslash A_{2}$ (where $\Gamma_{G}\left(A_{1}\right)$ is the neighborhood of $A_{1}$ in $G$ ). By the expansion property, we have:

$$
\left|\Gamma_{G}\left(A_{1}\right)\right| \geq\left|A_{1}\right|+d\left(1-\left|A_{1}\right| / n\right)\left|A_{1}\right| .
$$

In this case $d\left(1-\left|A_{1}\right| / n\right) \geq 2 k$. So $\left|\Gamma_{G}\left(A_{1}\right)\right|-\left|A_{1}\right| \geq 2 k$. So $\left|A_{2}\right|-\left|A_{1}\right| \geq k$. It implies $\varepsilon \geq 0$.

- $n-\frac{k}{d} \leq\left|A_{1}\right|$ :

we can assume that $\bar{Z}$ is connected (see the remarks on Proposition 1). As the vertices of $S$ are at distance at least $\alpha k$ with $\alpha k>\frac{k}{d}, \bar{Z}$ contains at most one vertex of $S$. Hence $i \geq\left|A_{2}\right|-k+1+\left|A_{1}\right|$ and $\varepsilon \geq \delta(Z)-\left|A_{2}\right|+\left|A_{1}\right|-1$. Because of the connectivity of $Z$ and the 3 -regularity $\left|A_{2}\right|>\left|A_{1}\right|$. So $\varepsilon \geq 0$.

\section{Graph Robustness: A New Approach to the De- sign of Valid Networks}

The construction of valid networks is related to a more general expansion property of graphs, more precisely to what can be seen as a 'bounded expansion property' or expansion property only for sets of bounded size. We call this property robustness.

Definition 3 ( $\alpha$-robustness) The $\alpha$-robustness, rob $_{\alpha}$, of a graph $G$ is the maximum value $r$, such that, for every subset $X \subset V(G)$ with $|X| \leq \frac{|V|}{2}$, we have $\delta(X) \geq$ $\min (r, \alpha|X|)$.

For $\alpha=1$ we simply say robustness. There are two main problems: on the one hand we want to understand the behavior of the robustness for a general graph, which means to give some upper bounds which can be done by showing the existence of subsets which violate the $\alpha$-robustness. This problem covers in particular the classical problem of bisection-width, which has several important applications, see for example [1] and [24]. On the other hand, for many applications, one would like to be able to find graphs of large robustness ( $\alpha$-robustness). The first examples of such graphs are expanders of expansion factor $c$ which give graphs with $c$-robustness at least $\frac{n}{2}$ where $n$ is the number of vertices. For $d$-regular graphs we have:

- if $G$ is $d$-connected, $r o b_{d}=1$ and $r o b_{d-1}=2$. $r o b_{d-2}=g$ where $g$ is the double girth of the graph (minimum size of a cycle with a chord); 
- for $\alpha \leq \frac{1}{2}(d-2 \sqrt{d-1}), \operatorname{rob}_{\alpha} \geq \frac{n}{2}$ for Ramanujan graphs;

- for $\alpha>\frac{d}{2}-c \sqrt{d}$, where $c$ is some absolute constant depending only on $d$, $\alpha$-robustness is strictly smaller than $\frac{n}{2}$ (see Alon [1]);

- for $\alpha>\frac{1}{3}+\epsilon$ the $\alpha$-robustness of a 3-regular graph is strictly smaller than $\frac{n}{2}$, the same is true for $\alpha>\frac{4}{5}+\epsilon$ and for 4-regular graphs (see Monien-Preis $[24])$.

Remark that, since it is NP-hard to compute the double girth of a graph, computing $\operatorname{rob}_{2}(G)$ is an NP-hard problem. The same is true for any $\alpha$.

Remark 1 Before going through the study of robustness we would like to stress that the concept of robustness is closely related to the concept of general selectors. A general selector is a selector without degree conditions. More precisely we have a graph $G$, such that the set of vertices $V(G)$ is partitioned into three subsets: $I$ the set of vertices with one input, $O$ the set of vertices with one output and $S$ the set of normal vertices with neither input nor output, with $|I|=p+k$ and $|O|=p$. We say that $G$ is a general selector (or is a $\left(\begin{array}{c}p+k \\ p\end{array}\right)$-network) if for any subset of $I$ of size $p$, there exist $p$ edge-disjoint paths, each one joining one different input to one different output.

Selectors also provide a very natural framework for constructing general valid networks, i.e., valid networks without degree constraints. For instance, for any $(p, \lambda, k)$, we can construct a general valid $(p, \lambda, k)$-network as follows: take two selectors, a $\left(\begin{array}{c}p+k \\ p\end{array}\right)$-network, $A$, and a $\left(\begin{array}{c}p+\lambda \\ p\end{array}\right)$-network, $B$. Let $G$ be the graph obtained by taking the disjoint union of $A$ and $B$, deleting the outputs connected to them, joining the two sets $O(A)$ and $O(B)$ by a matching and replacing the inputs connected to $I(B)$ by outputs. The resulting network is a general valid $(p, \lambda, k)$-network in which $I(A)$ is the set of vertices with one input and $I(B)$ is the set of vertices with one output.

In the following, $r_{\alpha}$ denotes the maximum $\alpha$-robustness of a 4-regular graph.

\subsection{Robustness of random 4-regular graphs}

In this section $\mathcal{G}(k, n)$ is the probability space of all graphs on $n$ vertices that are the union of $k$ disjoint Hamiltonian cycles, all graphs occurring with the same probability. By $G_{k, n}$ we denote an element of $\mathcal{G}(k, n)$. This probability space is "equivalent" to the probability space of random $2 k$-regular graphs, see [22].

Theorem 4 Random 4-regular graphs have 1-robustness at least $\frac{n}{14}$ with high probability, i.e. with probability $1-\mathcal{O}\left(\frac{\log n}{n}\right)$.

Corollary 1 For $k \leq \frac{n}{7}$, there exist $(p, \lambda, k)$-valid networks of size $3 n$ (remember that $n=p+k$ ).

Proof Suppose that a 4 -regular Hamiltonian graph $G$ of robustness $\frac{n}{7}$ on $2 n$ vertices is given. Extract a complete matching from a Hamiltonian cycle and add a doublon to every edge of this matching. It is now straightforward to show that the resulting network is valid. This gives a valid $(p=n-k, k, k)$-network with $3 n$ switches. 
Remark 2 The method of the proof of Theorem 4 can be also used to obtain lower bounds for $\alpha$-robustness of random 4-regular graphs for other values of $\alpha$. Some numerical results are given below for some special values of $\alpha$ :

- $r_{\frac{11}{25}}=\frac{n}{2}$ (See Bollobàs [15] for another proof of this result).

- $r_{\frac{11}{20}} \geq \frac{n}{2.6}, r_{\frac{4}{5}} \geq \frac{n}{5.7}, r_{\frac{2}{3}} \geq \frac{n}{3.6}, r_{\frac{3}{5}} \geq \frac{n}{3}$.

Proof [ of Theorem 4] We first give preliminary results on the unions of $k$ disjoint Hamiltonian cycles. The results for 4-regular graphs are then a direct application with $k=2$. Let $G$ be a graph of $\mathcal{G}(k, n)$ and $S$ be a subset of $V$ with $s$ vertices. On the cycles $C_{1}, C_{2}, \ldots C_{k}$ constituting $G, S$ can be described as a set of continuous intervals. For $i=1, \ldots k$, let $m_{i}(S)$ be the number of intervals that $S$ defines on $C_{i}$. Then $\delta(S)=2 \sum_{i=1,2, \ldots k} m_{i}(S)$.

For a given cyclic permutation $\pi$, if $I(s, m)$ denotes the number of sets of $s$ elements defining $m$ segments on the cycle associated to $\pi$, we have:

\section{Claim 1}

$I(s, m)=\left(\begin{array}{c}s-1 \\ m-1\end{array}\right)\left(\begin{array}{c}n-s \\ m\end{array}\right)+\left(\begin{array}{c}n-s-1 \\ m-1\end{array}\right)\left(\begin{array}{c}s \\ m\end{array}\right)=\left(\begin{array}{c}s \\ m\end{array}\right)\left(\begin{array}{c}n-s \\ m\end{array}\right)\left(\frac{m}{s}+\frac{m}{n-s}\right)$.

\section{Proof}

The number of ways to write a number $t$ as the ordered sum of $p$ non-negative integers is $\operatorname{cut}(t, p)=\left(\begin{array}{c}t+p-1 \\ p-1\end{array}\right)$. Also, the number of ways to write $t$ as the ordered sum of $p$ positive integers is $\operatorname{cut}(t-p, p)=\left(\begin{array}{c}t-1 \\ p-1\end{array}\right)$.

Starting from the vertex 0 of the cycle, the set $S$ can be encoded by giving two sequences of numbers : the ordered list of the lengths of the segments in $S$ and the ordered list of the lengths of the segments in $\bar{S}$. There are two cases, depending on wether the first interval belongs to $S$ or to $\bar{S}$.

- If 0 belongs to $\bar{S}$, then $S$ is associated with exactly $m$ segments on the path, all having length at least 1 , and $\bar{S}$ is associated to $m+1$ segments, all but the last one having length at least 1 (the last segment can have length 0 ).

So $\bar{S}$ can be encoded by dividing $|\bar{S}|-m=n-s-m$ into $m+1$ non-negative integers. There are exactly $\operatorname{cut}(n-s-m, m+1)$ ways of doing it.

We should also write $S$ as the ordered sum of $m$ non null numbers, there are $\operatorname{cut}(s-m, m)=\left(\begin{array}{c}s-1 \\ m-1\end{array}\right)$ possibilities.

This gives a contribution of $\operatorname{cut}(n-s-m, m+1) \cdot \operatorname{cut}(s-m, m)=\left(\begin{array}{c}s-1 \\ m-1\end{array}\right)\left(\begin{array}{c}n-s \\ m\end{array}\right)$ to $I(s, m)$.

- If 0 is in $S$, the situation is symmetric, with $n-s$ instead of $s$ : we replace $\operatorname{cut}(n-s-m, m+1) \operatorname{cut}(s-m, m)$ by $\operatorname{cut}(s-m, m+1) \operatorname{cut}(n-s-m, m)=$ $\left(\begin{array}{c}s \\ m\end{array}\right)\left(\begin{array}{c}n-s-1 \\ m-1\end{array}\right)$.

The total contribution is the sum of the partial contributions, and the claim follows.

Given a set $S$ with $s$ elements, we denote by $P(s, m)$ the probability that for a random cyclic permutation $\pi, S$ defines $m$ segments on the cycle associated to $\pi$. Since the probability does not depend on $S$, but only on $s, P(s, m)$ is well defined. We have: 
Claim 2

$$
P(s, m)=I(s, m) /\left(\begin{array}{c}
n \\
s
\end{array}\right)=\frac{\left(\begin{array}{c}
s \\
m
\end{array}\right)\left(\begin{array}{c}
n-s \\
m
\end{array}\right)\left(\frac{m}{s}+\frac{m}{n-s}\right)}{\left(\begin{array}{l}
n \\
s
\end{array}\right)} .
$$

Proof Consider a bipartite graph whose vertices are the sets of $s$ elements and the $(n-1)$ ! cyclic permutations. We put an edge between a set and a cyclic permutation if the set defines $m$ segments on the cycle associated to the permutation. This graph is a "regular bipartite graph", where sets have degree $\Delta$ and permutations have degree $I(s, m)$. Counting the edges of the graph, we get $\Delta=\frac{(n-1) ! I(s, m)}{\left(\begin{array}{c}n \\ s\end{array}\right)}$.

Now, the probability for a set to be adjacent to a permutation is $\Delta /(n-1)$ !. So, given a set $S$ with $s$ elements, the probability that $S$ defines exactly $m$ segments on a cycle $C$ is $I(s, m) /\left(\begin{array}{l}n \\ s\end{array}\right)$. Then Equation 1 gives the result.

\section{Claim 3}

$$
\operatorname{Prob}(\delta(S)=2 x)=\sum_{k_{1}, \ldots, k_{k} \mid k_{1}+k_{2}+\ldots+k_{k}=x} \prod I\left(s, k_{i}\right) /\left(\begin{array}{l}
n \\
s
\end{array}\right)^{k} .
$$

Proof The result follows from the three points below:

- the probability that $S$ defines $k_{i}$ segments on a random cycle $C_{i}$ is $I\left(s, k_{i}\right)$;

- the cycles constituting $G_{k, n}$ are independent

- $\delta(S)=2 \sum_{1,2, \ldots, k} k_{i}$.

We say that a set is bad if $\delta(S)<\min (s, n-s, r)$. By the above equations we can estimate the probability of having bad sets. The following two lemmas complete the proof of our Theorem.

Lemma 2 (Robustness for small sets) Any set with size $s \leq \frac{n}{14}$ has border at least $s$ with probability $1-\mathcal{O}\left(\frac{\log n}{n}\right)$.

Proof

The number of cycles is two for 4-regular graphs. Let $W(n, s)$ be the probability that there is a set of size $s \leq r=\frac{n}{14}$ with border less than $s$ :

$$
W(n, s) \leq\left(\begin{array}{l}
n \\
s
\end{array}\right) \sum_{r \leq s / 2} \sum_{m_{1}+m_{2}=r} I\left(s, m_{1}\right) I\left(s, m_{2}\right) /\left(\begin{array}{l}
n \\
s
\end{array}\right)^{2} .
$$

Since $I\left(s, m_{1}\right) I\left(s, m_{2}\right) \leq I\left(s, \frac{m_{1}+m_{2}}{2}\right)$, we have

$$
W(n, s)=\sum_{r \leq s / 2} \sum_{m_{1}+m_{2}=r} I\left(s, m_{1}\right) I\left(s, m_{2}\right) /\left(\begin{array}{l}
n \\
s
\end{array}\right) \leq \sum_{r \leq s / 2} r(I(s, r / 2))^{2} /\left(\begin{array}{l}
n \\
s
\end{array}\right) .
$$

Now, since $r \leq s / 2$, we have $I(s, r / 2) \leq I(s, s / 4)$ and $W(n, s) \leq \frac{s^{2}}{8}(I(s, s / 4))^{2} /\left(\begin{array}{l}n \\ s\end{array}\right)$. Replacing $I(s, s / 4)$ by its value we get:

$$
W(n, s) \leq \frac{s^{2}}{8}\left(\begin{array}{c}
s \\
s / 4
\end{array}\right)^{2}\left(\begin{array}{c}
n-s \\
s / 4
\end{array}\right)^{2} /\left(\begin{array}{l}
n \\
s
\end{array}\right) .
$$


For the following we need the following rather sharp form of Stirling's formula proved by Robbins(1955):

$$
n !=\left(\frac{n}{e}\right)^{n} \sqrt{2 \pi n} e^{\alpha_{n}},
$$

where $\frac{1}{12 n+1}<\alpha_{n}<\frac{1}{12 n}$.

Putting this formula in the above inequality we obtain:

$$
W(n, s) \leq \frac{s^{3 s}(n-s)^{3 n-3 s}}{\left(\frac{s}{4}\right)^{s}\left(\frac{3 s}{4}\right)^{\frac{3 s}{2}}\left(n-\frac{5 s}{4}\right)^{2 n-\frac{5 s}{2}} n^{n}} \beta,
$$

where $\beta=\frac{16(n-s) \sqrt{s(n-s)}}{3 \pi \sqrt{2 \pi n}(4 n-5 s)} \cdot e^{\gamma}$ with

$$
\gamma=3 \alpha_{s}+3 \alpha_{n-s}-4 \alpha_{\frac{s}{4}}-2 \alpha_{\frac{3 s}{4}}-2 \alpha_{n-\frac{5 s}{4}}-\alpha_{n},
$$

$\alpha_{i}$ being a real number in the interval $\left[\frac{1}{12 i+1}, \frac{1}{12 i}\right]$. Let

$$
X(n, s):=\frac{s^{3 s}(n-s)^{3 n-3 s}}{\left(\frac{s}{4}\right)^{s}\left(\frac{3 s}{4}\right)^{\frac{3 s}{2}}\left(n-\frac{5 s}{4}\right)^{2 n-\frac{5 s}{2}} n^{n}} \beta .
$$

Suppose $n=a \cdot s$. Then we have:

$$
X(n, s)=\left(\frac{(a-1)^{3(a-1)}}{\frac{1}{4} \cdot\left(\frac{3}{4}\right)^{\frac{3}{2}} \cdot\left(a-\frac{5}{4}\right)^{2 a-\frac{5}{2}} \cdot a^{a}}\right)^{s} \beta .
$$

If we note

$$
g(a):=\frac{(a-1)^{3(a-1)}}{\frac{1}{4} \cdot\left(\frac{3}{4}\right)^{\frac{3}{2}} \cdot\left(a-\frac{5}{4}\right)^{2 a-\frac{5}{2}} \cdot a^{a}},
$$

we have

$$
X(n, s)=g(a)^{s} \cdot \beta .
$$

Remember that

$$
\beta=\frac{16(n-s) \sqrt{s(n-s)}}{3 \pi \sqrt{2 \pi n}(4 n-5 s)} \cdot e^{\gamma} .
$$

It is now easy to see that $g$ is decreasing. As $g(13.80 \cdots)=1$, we have $g(14)<1$. Hence

- For $s \in\left[-\frac{4 \log n}{\log (g(14))}, \frac{n}{14}\right]$ we have

$$
X(n, s) \leq C \cdot \frac{1}{n^{4}} \beta=O\left(\frac{1}{n^{2}}\right),
$$

for some constant $C$. Hence for some other constant $C$,

$$
W(n, s) \leq C \cdot \frac{1}{n^{2}} \text {. }
$$

- For $s \leq-\frac{4 \log n}{\log (g(14))}$ we have $a \geq C \cdot \frac{n}{\log n}$ (for some constant $C$ ). Hence

$$
X(n, s) \leq C .(n / s)^{-\frac{s}{2}} \cdot \beta .
$$

It is clear that for $s \geq 4$, we have $X(n, s) \leq C \cdot \frac{1}{n}$ for some constant $C$. 
- It is easy to show that $W(n, s)=0$ for $s=1,2,3$, as the edge connectivity of a random 4-regular graph is 4 with very high probability.

Then we have

$$
\begin{array}{r}
\sum_{s \leq \frac{n}{14}} W(n, s) \leq \sum_{-\frac{4 \log n}{\log (g(14))} \leq s \leq \frac{n}{14}} X(n, s)+\sum_{s \leq-\frac{4 \log n}{\log (g(14))}} X(n, s) \\
<\text { C.n. } \frac{1}{n^{2}}+\frac{C \cdot \log (n)}{n}=\mathcal{O}\left(\frac{\log n}{n}\right) .
\end{array}
$$

Lemma 3 (Robustness for large sets) For $n$ large enough, any set of size $s \in$ $\left[\frac{n}{14}, \frac{13 n}{14}\right]$ has border at least $\frac{n}{14}$ with probability $1-\mathcal{O}\left(\frac{1}{n}\right)$.

\section{Proof}

A set larger than $\frac{n}{14}$ is bad when its border is smaller than $\frac{n}{14}$. Let $W^{\prime}(n, s)$ be the probability that there exists a set of size $s \geq r=\frac{n}{14}$ with border less than $r=\frac{n}{14}$. We have

$$
W^{\prime}(n, s) \leq \sum_{i \leq \frac{n}{28}} \sum_{k_{1}+k_{2}=i} I\left(s, k_{1}\right) I\left(s, k_{2}\right) /\left(\begin{array}{l}
n \\
s
\end{array}\right) .
$$

Hence $W^{\prime}(n, s) \leq \frac{\left(\frac{n}{14}\right)^{2}}{8}\left(I\left(s, \frac{n}{56}\right)\right)^{2} /\left(\begin{array}{l}n \\ s\end{array}\right)$.

Let define $\delta(a)$ for $a \in\left[\frac{1}{14}, 1-\frac{1}{14}\right]$ as

$$
\delta(a)=\frac{\left.\left(\begin{array}{c}
a \cdot n \\
\frac{n}{56}
\end{array}\right)\left(\begin{array}{c}
(1-a) n \\
\frac{n}{56}
\end{array}\right)\right)^{2}}{\left(\begin{array}{c}
n \\
. . n
\end{array}\right)} .
$$

Then

$$
W^{\prime}(n, a n) \leq \frac{1}{8}\left(\frac{n}{14}\right)^{2} \delta(a)
$$

Again, using methods similar to the ones of last section, we obtain $W^{\prime}(n, a) \leq$ $h(a)^{n} \beta^{\prime}$, where $\beta^{\prime}$ is a rational function on $n$ and $a$, and $h$ is defined below:

$$
h(a)=\frac{a^{3 a}(1-a)^{3-3 a}}{\left(\frac{1}{56}\right)^{\frac{1}{14}}\left(a-\frac{1}{56}\right)^{2 a-\frac{1}{28}}\left(1-a-\frac{1}{56}\right)^{2-2 a-\frac{1}{28}}} \beta^{\prime} .
$$

Since $h\left(\frac{1}{14}\right)<1$, with $h$ decreasing,

$$
W^{\prime}(n, a n) \leq h\left(\frac{1}{14}\right)^{n} \beta^{\prime} .
$$

As $\beta^{\prime}$ is rational, we have for $n$ large enough $W^{\prime}(n, a n)<\frac{1}{2 n^{2}}$, and so $W^{\prime}(n, s)=$ $\mathcal{O}\left(\frac{1}{n}\right)$. So

$$
\sum_{s \in\left[\frac{n}{14}, \frac{13 n}{14}\right]} W^{\prime}(n, s)=\mathcal{O}\left(\frac{1}{n}\right),
$$

and the lemma is proved. 


\section{Lower Bounds}

In this section we distinguish switches according to their number of inputs and outputs. Figure 4 defines the different set of switches: $S, S_{i}, S_{o}, V_{i}, V_{o}, D$ and $T$. For example, a switch $v$ is in $D$ if $i(v)=o(v)=1$. Remember that we called such a switch a doublon and that all switches that are not a doublon are called $R$-switch. Remark that direct applications of the cut criterion show that no other types of switches are possible and that, as soon as $\lambda \geq 1$, switches of kind $T$ are forbidden. Recall the convention that a lower case letter in the notations indicates the cardinality of the set denoted by the corresponding upper case letter.

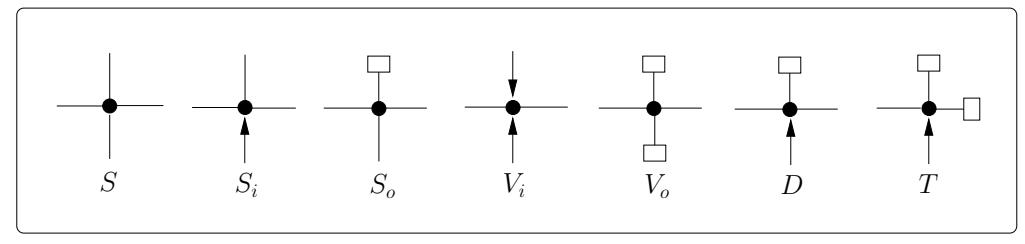

Figure 4: Kinds of switches.

Fundamental equations are linking the different kinds of switches: Equation 4 (switch partition equation) which counts the number $N$ of switches of the network, Equation 5 (input equation) which counts the number of inputs and Equation 6 (output equation) which counts the number of outputs.

$$
\begin{aligned}
N & =s+s_{i}+s_{o}+d+v_{i}+v_{o}+t \\
p+\lambda & =s_{i}+2 v_{i}+d+t \\
p+k & =s_{o}+2 v_{o}+d+2 t
\end{aligned}
$$

In Subsection 6.1, we prove a fundamental preliminary theorem, Theorem 5 . Its main point is that $N(p, \lambda, k) \geq \frac{3}{2} n+\frac{d}{2}-\varepsilon(n)$ (where $d$ is the number of doublons and where $\varepsilon(n)$ goes to zero when $n$ goes to infinity). Direct applications of this theorem give lower bounds for general and simplified networks (Theorems 6 and $7)$. We succeed in obtaining a better bound of $n+\frac{2}{3} n-\varepsilon(n)$ for two cases: when $\lambda$ goes to infinity (Theorem 8) and when some kinds of switches are not allowed in the networks (Theorem 9). Finally (Theorem 10), we succeed to obtain a tight bound $n+\frac{3}{4} n-\varepsilon(n)$ for another family of networks using majority arguments.

\subsection{Preliminary Theorem}

The idea to find and prove lower bounds is first to obtain local information on possible switch configurations. The methodology is to use the cut criterion (Proposition 1) to infer equations linking the numbers of switches of different kinds. Then, we use the existence of a quasi-partition (Lemma 4) to obtain bounds for the whole network.

Definition 4 (q-quasi-partition) Let $G=(V, E)$ be a graph and $q$ be a positive integer. A q-quasi-partition of $G$ is a family $Q=\left\{A_{1}, A_{2}, \ldots, A_{m}\right\}$ of subsets of $V$, such that : 
(i) for every $1 \leq i \leq m$, the subgraph $G\left[A_{i}\right]$ induced by $A_{i}$ is connected;

(ii) for every $1 \leq i \leq m, \frac{q}{3} \leq\left|A_{i}\right| \leq q$;

(iii) $V=\bigcup_{i=1}^{m} A_{i}$ and $\sum_{i=1}^{m}\left|A_{i}\right| \leq|V|+\left|\left\{A_{i} ;\left|A_{i}\right|>\frac{2 q}{3}\right\}\right|+1$.

Lemma 4 ([20]) Let $q$ be a positive integer and $G$ be a connected graph of order at least $\frac{q}{3}$. Then $G$ admits a q-quasi-partition.

Remark 3 - If $G$ has several connected components, each of size at least $\frac{q}{2}$, applying the lemma to each component and using the additivity of both sides of Equation (iii) gives us a $q$-quasi-partition of $G$.

- Let $Q$ be a quasi-partition of $G$ as in Lemma 4. Let $t=\left|\left\{A_{i},\left|A_{i}\right| \geq \frac{2 q}{3}\right\}\right|$ and $v=|V|$. Then we have: $m \leq \frac{3(v+t+1)}{q}$ and $t \leq \frac{v+1}{\frac{2 q}{3}-1}$.

Proof The lemma as stated in [20] is slightly incorrect. Hence we restate it here and give a new proof. Since every connected graph $G$ contains a spanning tree, it is sufficient to prove the result for trees. We prove this by induction on the size of the trees. Let $T$ be a tree. If $T$ contains less than $q$ elements, then the result trivially holds. So suppose that $|T| \geq q$. An edge $e$ of $T$ is called a $q$-balanced separator, if removing $e$ separates the tree into connected components of sizes at least $q$. Let $E_{q}$ be the subgraph consisting of all $q$-balanced edges. If there is no $q$-balanced edge in the tree, a q-separator vertex exists, that is a vertex $v$ such that all the connected components of $T \backslash\{v\}$ have less than $q$ vertices. Let us take $l$ to be a leaf of $E_{q}$ if some $q$-balanced edges exist or to be a $q$-separator vertex if $E_{q}=\emptyset . T \backslash\left(E_{q} \cup\{l\}\right)$ has $r$ connected components, say $C_{1}, \ldots, C_{r}$, such that $\left|C_{1}\right| \geq\left|C_{2}\right| \geq \cdots \geq\left|C_{r}\right|$. By the choice of $l$, and our hypothesis on the size of $T(|T|>q)$, we have $\left|C_{i}\right|<q$ and $\sum_{i=1}^{r}\left|C_{i}\right| \geq q$. The following cases can appear.

- $\left|C_{1}\right| \geq \frac{q}{3}$ and $T \backslash C_{1}$ has at least $\frac{q}{3}$ vertices. Then by induction it has a $q$ quasi partition $\left\{A_{1}, \ldots, A_{m-1}\right\}$. Adding $A_{m}=V\left(C_{1}\right)$ to this family provides a $q$-quasi partition for $T$.

- $\left|C_{1}\right|<\frac{q}{3}$. We have $r \geq 3$. Let $s$ be the largest integer such that $\sum_{i \leq s}\left|C_{i}\right| \leq q$. As $\left|C_{s+1}\right| \leq\left|C_{1}\right|<\frac{q}{3}$, we have $s \geq 3$ and $\sum_{i \leq r}\left|C_{i}\right| \geq \frac{2 q}{3}$ (by maximality of $s)$.

So we can suppose that $\sum_{i \leq r}\left|C_{i}\right| \geq \frac{2 q}{3}$. If the connected tree $T \backslash \bigcup_{i \leq s} C_{i}$ has more than $\frac{q}{3}$ elements, for example if $E_{q} \neq \emptyset$, by induction it admits a $q$-quasi partition $\left\{A_{1}, \ldots, A_{m-1}\right\}$. Adding $A_{m}=\bigcup_{i \leq s} C_{i} \cup\{l\}$ to this family provides a $q$-quasi partition for $T$.

- $T$ has less than $q+\frac{q}{3}$ vertices, $E_{q}$ is empty and we can find a vertex $l$ such that each $\left|C_{i}\right|$ has less than $\frac{q}{3}$ vertices. Let $t$ be the smallest integer such that $\sum_{i \leq t}\left|C_{i}\right| \geq \frac{q}{3}$. We claim that $T \backslash \bigcup_{i \leq t} C_{i}$ has at most $q$ and at least $\frac{q}{3}$ vertices. The first one is true because $T$ has at most $q+\frac{q}{3}$ vertices and $\bigcup_{i<t} C_{i}$ has at least $\frac{q}{3}$ vertices. The second one is true, because of the minimality of $t$. Indeed, $\bigcup_{i<t-1} C_{i}$ has at most $\frac{q}{3}$ vertices, $C_{t}$ has at most $\frac{q}{3}$ vertices, and so because $|T| \geq q+1, T \backslash \bigcup_{i \leq t} C_{i}$ has at least $\frac{q}{3}$ vertices. It is now easy to check that $\bigcup_{i \leq t} C_{i} \cup\{l\}$ and $T \backslash \bigcup_{i \leq t} C_{i}$ form a q-quasi partition of $T$. 
For the proof of Theorem 5 we need to define large and small $H$-components of $\mathcal{R}$, the associated graph of $\mathcal{N}$.

Definition 5 [ $H$-component, large and small $H$-components, adjacent $H$ components] We consider a $(p, \lambda, k)$-network and its associated graph $\mathcal{R}$ (see Section 2 for definition). We take $H$ the subgraph of $\mathcal{R}$ which contains only the edges of type $E_{0} . A n H$-component of $\mathcal{R}$ is a connected component of $H$. An $H$-component is said large (respectively small) if it has more than (resp. strictly less than) $q$ switches, with $q$ the greatest integer satisfying $2(q+(2 q+2) q)+2 \leq k-1$. Remark that $q \sim \frac{\sqrt{k}}{2}$. Two $H$-components $C_{1}$ and $C_{2}$ are said adjacent if there exists an edge of $\mathcal{R}$ with one $R$-switch in $C_{1}$ and the other one in $C_{2}$.

Proposition 2 1. A small $H$-component has no outgoing edge of kind $E_{2}$.

2. A small H-component has no input inside.

3. Two small H-components are not adjacent.

Proof Let $C$ be a small $H$-component. We apply the cut criterion of Section 4.2 to the set $\tilde{C}$ of $\mathcal{N}$ obtained from $C$ by adding in $\mathcal{N}$ the doublons of the edges of type $E_{1}$ and $E_{2}$ incident to $R$-switches of $C$.

As $k \leq p$ and $|C| \leq q \approx \frac{\sqrt{k}}{2}$, we have $o(C) \leq \frac{k}{2} \leq p$. Hence the cut criterion reduces to

$$
\delta(\tilde{C}) \geq i(\tilde{C}) .
$$

Let $e_{1}$ (resp. $e_{2}$ ) be the number of outgoing edges of kind $E_{1}$ (resp. $E_{2}$ ) incident to $R$-switches of $C$.

- By definition of $\mathrm{H}$ and of the small components, $\delta(\tilde{C})=e_{1}+e_{2}$. We have $i(\tilde{C}) \geq e_{1}+2 e_{2}+i(C)$. So by the cut criterion, $e_{2}=0$ proving point 1$)$ and $i(C)=0$ proving point 2 ).

- Let $C^{\prime}$ be an other small $H$-component. If $C$ and $C^{\prime}$ are joined by $f \geq 1$ edges, then let $W=\tilde{C} \cup \tilde{C}^{\prime}$, we have $i(W) \geq e_{1}+e_{1}^{\prime}-f$ and $\delta(W) \leq e_{1}+e_{1}^{\prime}-2 f$ so $\delta(W)<i(W)$, which gives a contradiction.

Theorem 5 (Preliminary Theorem) In a valid network $\mathcal{N}$ with $k \leq \frac{n}{2}$, we have

$$
\begin{aligned}
N & \geq\left(\frac{3}{2} n-(k-\lambda)-4-\frac{12}{\sqrt{k}}\right)\left(1-\frac{9}{2 \sqrt{k}}+O\left(\frac{1}{k}\right)\right) \\
& +\frac{d}{2}\left(1+\frac{9}{2 \sqrt{k}}+O\left(\frac{1}{k}\right)\right),
\end{aligned}
$$

where $n=p+k$.

Proof According to Lemma 4 and Remark 3, the union of the large $H$-components of $\mathcal{R}$ admits a $q$-quasi-partition $Q=\left\{A_{1}, \ldots, A_{m}\right\}$. So each $A_{j}$ is connected and of size $\frac{q}{2} \leq\left|A_{j}\right| \leq q$. We say that an edge with doublons (i.e. an edge of type $E_{1}$ or $E_{2}$ ) is of type: 
- $R$ if it is between an $A_{j}$ and a small $H$-component,

- $M$ if it is between two distinct sets: $A_{j}$ and $A_{k}$,

- $N$ if it is inside an $A_{j}$.

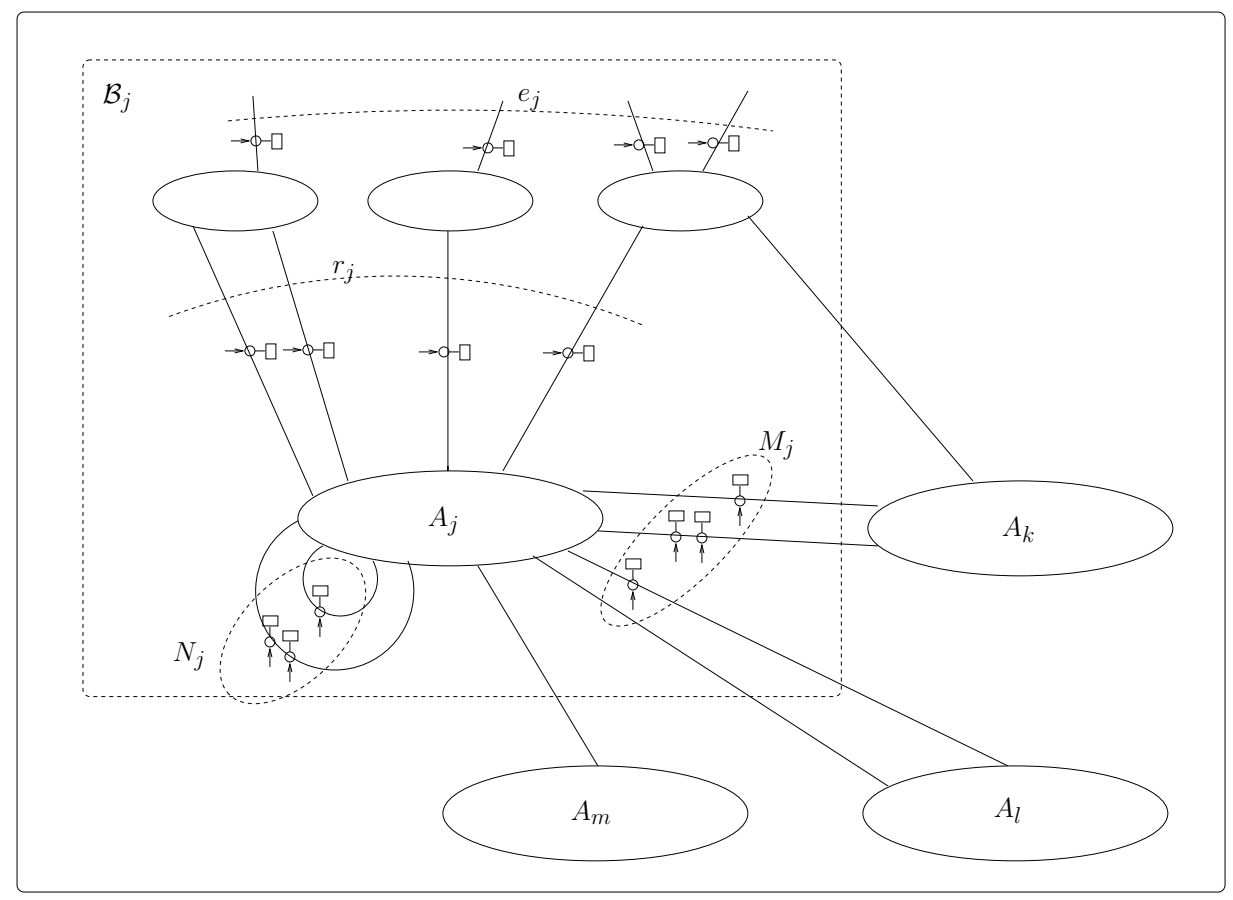

Figure 5: Sketch of proof of Theorem 5

For each $A_{j}$, we introduce the set $\mathcal{B}_{j}$ consisting of $A_{j}$ and all the small $H$-components adjacent to it. We define new quantities as shown on Figure 5:

- $r_{j}$ is the number of edges between $A_{j}$ and its small H-components.

- $e_{j}$ is the number of outgoing edges of $\mathcal{B}_{j}$ incident to one of its small components.

- $M_{j}$ is the number of doublons on the edges of type $M$.

- $N_{j}$ is the number of doublons on the edges of type $N$.

As two small components can not be adjacent, remark that all $r_{j}$ and $e_{j}$ edges are of type $R$.

Let us now apply the cut criterion to $\mathcal{B}_{j} . \quad \delta\left(\mathcal{B}_{j}\right)+o\left(\mathcal{B}_{j}\right)-\min \left(k, o\left(\mathcal{B}_{j}\right)\right)-$ $\min \left(i\left(\mathcal{B}_{j}\right), p\right) \geq 0$. As $k \leq \frac{n}{2}$, we have $i\left(\mathcal{B}_{j}\right) \leq p$. We first show that $o\left(\mathcal{B}_{j}\right)<k$. Indeed, if $o\left(\mathcal{B}_{j}\right) \geq k$, the cut criterion reduces to $\delta\left(\mathcal{B}_{j}\right) \geq k$. Furthermore $A_{j}$ is connected and of size less than $q$, so it has at most $2 q+2$ outgoing edges and the number of small $H$-components of $\mathcal{B}_{j}$ is at most $2 q+2$. As the size of a small $H$-component is less than $q$, the number of vertices in $\mathcal{B}_{j}$ is at most $q+(2 q+2) q$. 
Hence the number of outgoing edges $\delta\left(\mathcal{B}_{j}\right)$ is at most $2(q+(2 q+2) q)+2$. By our choice of $q$ it gives $k \leq \delta\left(\mathcal{B}_{j}\right) \leq 2(q+(2 q+2) q)+2 \leq k-1$, a contradiction. Consequently $o\left(\mathcal{B}_{j}\right)<k$.

The cut criterion is now equivalent to $\delta\left(\mathcal{B}_{j}\right) \geq i\left(\mathcal{B}_{j}\right)$. Noting $\delta^{\prime}$ the number of outgoing edges of $\mathcal{B}_{j}$ incident to $A_{j}$, we have $\delta\left(\mathcal{B}_{j}\right)=\delta^{\prime}\left(\mathcal{B}_{j}\right)+e_{j}$. Using the definitions of switch kinds (Figure 4), we have

$$
\delta^{\prime}=4\left|A_{j}\right|-2 e\left(A_{j}\right)-r_{j}-2 N_{j}-s_{i}-s_{o}-2 v_{i}-2 v_{o}-3 t .
$$

Since $A_{j}$ is connected, $e\left(A_{j}\right) \geq\left|A_{j}\right|-1$, we get

$$
\delta^{\prime} \leq 2\left|A_{j}\right|+2-r_{j}-2 N_{j}-s_{i}-s_{o}-2 v_{i}-2 v_{o}-3 t .
$$

For the number of inputs in $\mathcal{B}_{j}$ we have

$$
i\left(\mathcal{B}_{j}\right)=e_{j}+r_{j}+M_{j}+N_{j}+s_{i}+2 v_{i}+t .
$$

The cut criterion (Proposition 1) then implies:

$$
2\left|A_{j}\right|+2 \geq 2 s_{i}+s_{o}+4 v_{i}+2 v_{o}+4 t+3 N_{j}+2 r_{j}+M_{j} .
$$

The total number of doublons is $d$. All doublons are of type $R, M$ or $N$. The doublons in $\mathrm{M}$ are counted for two different $A_{j}$ and $A_{k}$. So $\sum_{j=1}^{m} M_{j}+3 N_{j}+2 r_{j} \geq$ $2 d$. Hence taking the sum of Equation 7 over all $j$ we obtain

$$
2 \sum_{j=1}^{m}\left|A_{j}\right|+2 m \geq 2 s_{i}+s_{o}+4 v_{i}+2 v_{o}+4 t+2 d .
$$

The input and output equations (Equations 5 and 6) give $2 s_{i}+4 v_{i}+2 t+2 d=$ $2 n-2(k-\lambda)$ and $s_{o}+2 v_{o}+2 t=n-d$. Hence

$$
2 \sum_{j=1}^{m}\left|A_{j}\right|+2 m \geq 3 n-d-2(k-\lambda)
$$

The family $\left\{A_{j}\right\}$ forms a $q$-quasi-partition of $\mathcal{R}$. Let $t:=\left|\left\{A_{j},\left|A_{j}\right| \geq \frac{2 q}{3}\right\}\right|$ and $v$ the number of vertices of $\mathcal{R}$. Then, by Remark 3 , we have $m \leq \frac{3(v+t+1)}{q}$ and $t \leq \frac{v+1}{\frac{2 q}{3}-1}$. By definition of a quasi-partition: $\sum_{i=j}^{m}\left|A_{j}\right| \leq v+\left|\left\{A_{j},\left|A_{j}\right| \geq \frac{2 q}{3}\right\}\right|=v+t+1$.

Putting all these equations into Equation 8 gives:

$$
\begin{gathered}
2 v\left(1+\frac{1}{\frac{2 q}{3}-1}\right) \geq \frac{q}{q+3}\left[(3 n-d-2(k-\lambda))-\frac{2(q+3)}{q}-\frac{6}{q}-2\right] . \\
2 v \geq \frac{q}{q+3} \frac{2 q-3}{2 q}\left[3 n-d-2(k-\lambda)-\frac{2(q+3)}{q}-\frac{6}{q}-2\right] \\
=\left[3 n-d-2(k-\lambda)-\frac{2(q+3)}{q}-\frac{6}{q}-2\right]\left(1-\frac{9}{2 q+6}\right) .
\end{gathered}
$$

Using $N \geq v+d$, we obtain

$$
N \geq\left(\frac{3}{2} n-(k-\lambda)-4-\frac{12}{q}\right)\left(1-\frac{9}{2 q+6}\right)+\frac{d}{2}\left(1+\frac{9}{2 q+6}\right) .
$$


Finally,

$$
\begin{aligned}
N & \geq\left(\frac{3}{2} n-(k-\lambda)-4-\frac{12}{\sqrt{k}}\right)\left(1-\frac{9}{2 \sqrt{k}}+O\left(\frac{1}{k}\right)\right) \\
& +\frac{d}{2}\left(1+\frac{9}{2 \sqrt{k}}+O\left(\frac{1}{k}\right)\right) .
\end{aligned}
$$

\subsection{Lower Bounds}

Here we apply Theorem 5 to prove lower bounds for the number of switches of minimal valid networks. Let us remind the reader that $l \leq k$ is assumed for symmetry reasons and that $n:=p+k$.

Theorem 6 (Selector: $\lambda=0$ ) In a valid network $\mathcal{N}$, when $k \rightarrow \infty$ with $k \leq \frac{n}{2}$, we have

$$
N \geq n+\frac{n}{2}+\mathcal{O}\left(\frac{n}{\sqrt{k}}\right) .
$$

In other words,

$$
N(p, 0, k) \geq n+\frac{n}{2}+O\left(\frac{n}{\sqrt{k}}\right) .
$$

In particular, we obtain a tight bound for networks with $\lambda=0$ (see Theorem 3).

Proof The proof follows directly from Theorem 5 .

Theorem 7 (Simplified case) In the simplified case, when $k \rightarrow \infty$ with $k \leq \frac{n}{2}$, we have $N(p, \lambda, k) \geq 2 n+O\left(\frac{n}{\sqrt{k}}\right)$.

In particular, we obtain a tight bound for the simplified case (see Theorem 1).

Proof First recall that a direct application of the cut criterion shows that as soon as $\lambda \geq 1, T$ is empty (see definition of switch types in Figure 4, p. 18). This means that in this proof and the following we will not consider switches of $T$. Now the proof follows from Theorem 5 and from $d=n-(k-\lambda)$ in the simplified case.

Theorem 8 (General case) When $\lambda \rightarrow \infty$ and $k \rightarrow \infty, N(p, \lambda, k) \geq n+\frac{2}{3} n+$ $O\left(\frac{n}{\sqrt{\lambda}}\right)$.

Proof We first give a bound for the number of switches of types $V_{i}$ and $V_{o}$ using the following Lemma:

Lemma 5 When $\lambda \rightarrow \infty$ and $k \rightarrow \infty$,

$$
\left\{\begin{array}{l}
v_{i} \leq N-\frac{3}{2} n-\frac{d}{2}-\frac{k-\lambda}{2}+\lambda O\left(\frac{n}{\sqrt{k}}\right), \\
v_{o} \leq N-\frac{3}{2}(n-k+\lambda)-\frac{d}{2}+k-\frac{\lambda-k}{2}+O\left(\frac{n}{\sqrt{\lambda}}\right) .
\end{array}\right.
$$



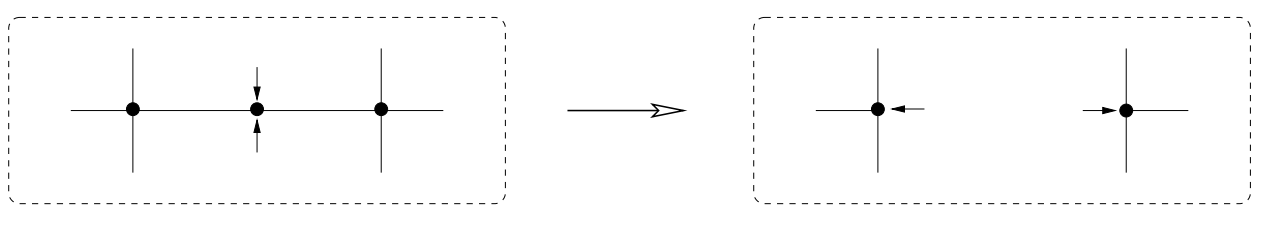

Figure 6: When $\lambda=0$, switches of kind $V_{i}$ may be removed.

Proof Imagine we have a valid $(p, \lambda, k)$-network with $N$ switches and $v_{i}$ switches in $V_{i}$. We obtain a valid $(p, 0, k)$-network after removing any $\lambda$ inputs. This new network has at least $v_{i}-\lambda$ switches of kind $V_{i}$. By Remark 4 we may remove these switches and obtain a valid $(p, 0, k)$-network with $N-v_{i}+\lambda$ switches. Theorem 5 gives

$$
N-v_{i}+\lambda \geq \frac{3}{2} n+\frac{d}{2}+\frac{k-\lambda}{2}+O\left(\frac{n}{\sqrt{k}}\right) .
$$

So the result holds. Symmetry (in the sense of swapping inputs and outputs gives a valid $(p, k, \lambda)$-network) gives the second equation.

The switch partition equation and Equations 5 and 6 give here

$$
N=2 n-(k-\lambda)-v_{i}-v_{o}-d+s .
$$

Lemma 5 gives

$$
\begin{aligned}
N \geq & 2 n-(k-\lambda)-d+s-2 N+3 n+d \\
& \quad+O\left(\frac{n}{\sqrt{k}}\right)+O\left(\frac{n}{\sqrt{\lambda}}\right)-\frac{3}{2}(k-\lambda)-\lambda-k . \\
N \geq & \frac{5}{3} n+O\left(\frac{n}{\sqrt{k}}\right)+O\left(\frac{n}{\sqrt{\lambda}}\right)-\frac{5}{2} k+\frac{\lambda}{2} .
\end{aligned}
$$

Theorem 9 When $\lambda \geq 1$ and no switches of kinds $V_{i}$ and $V_{o}$ are allowed, when $k \rightarrow \infty$ with $k \leq \frac{n}{2}$, we have $N(p, \lambda, k) \geq n+\frac{2}{3} n+O\left(\frac{n}{\sqrt{k}}\right)$.

Proof Let us first remark the following:

Remark 4 Notice that when $\lambda=0$, switches of type $V_{i}$ are not present in a minimal valid $(p, \lambda, k)$-network. As shown in Figure 6 , they may be removed to form a new valid $(p, \lambda, k)$-network with $v_{i}$ less switches.

Proof Direct by the cut criterion.

When $v_{i}=v_{o}=0$, the input equation (Equation 5) becomes $n=d+s_{i}$ and the output equation (Equation 6) becomes $n=d+s_{o}$. So $s_{i}=s_{o}$ and the switch partition equation (Equation 4) gives

$$
N=2 n-d .
$$

Theorem 5 gives

$$
2 n-d \geq \frac{3}{2} n+\frac{d}{2}+O\left(\frac{n}{\sqrt{k}}\right)
$$




$$
\frac{n}{3} \geq d+O\left(\frac{n}{\sqrt{k}}\right)
$$

Equation 9 gives

$$
N \geq \frac{5}{3} n+O\left(\frac{n}{\sqrt{k}}\right)
$$

To sum up, in the general case, we proved a lower bound of type $n+2 n / 3$. Let us remind the reader that we obtained an upper bound with $n+3 n / 4$ switches (Theorem 2). We conjecture that the later is the right answer to the problem. We are able to prove this with some extra conditions on the network using majority arguments:

Theorem 10 Let $\mathcal{N}$ be a network of $N$ switches with the associated graph $\mathcal{R}=$ $(V=A \cup B, E)$ such that $\mathcal{R}$ is bipartite, all vertices of $A$ have exactly one output and all vertices of $B$ exactly one input in the original network.

$$
N \geq n+\frac{3}{4} n+O\left(\frac{n}{\sqrt{k}}\right) .
$$

Proof We are given a ternary bipartite graph $R=(V=A \cup B, E)$ such that all vertices of $A$ have an output and all vertices of $B$ have an input. The vertices of $B$ are partitioned in two sets $B_{1}$ and $B_{0}$. A vertex of $B_{1}$ is adjacent to an edge of type $E_{1}$ or $E_{2}$, while the vertices of $B_{0}$ are not.

For a subset $X \subset A$ we use the following notations : $B_{i}(X)=\Gamma(X) \cap B_{i}, i=0,1$ and $b_{i}=\left|B_{i}\right|$.

Let $X$ be a subset of $A$ such that $F(X)=X \cup \Gamma(X)$ is connected and $6|X| \leq k$. $X$ has less than $k$ outputs. To fullfill the cut criterion for small subsets (less than $k$ outputs), we need

$$
b_{0}(X) \geq b_{1}(X)-3+3 c_{F(X)},
$$

where $c_{F(X)}$ is the feedback edge set of the subgraph induced by $F(X)$.

The goal is to prove that this can happen only if $b_{0}+O\left(\frac{1}{\sqrt{k}}\right) \geq 2 b_{1}$.

Let $Y$ be a set of $y$ vertices of $B_{0}$ such that $Y \cup \Gamma(Y)$ is connected. We have $|\Gamma(Y)|=2 y+1-c_{F(Y)}$. There are $3 y+3-3 c$ edges comming out of $F(Y)$, say $\alpha y$ toward vertices of type $B_{0}$ and $3 y+3-3 c-\alpha y$ toward vertices of type $B_{1}$. In $G$ we consider the connected subgraph induced by $Z=F(Y) \cup\left(\Gamma(F(Y)) \cap B_{1}\right)$. We have $(6-\alpha) y+3-3 c_{F(Y)}$ edges inside $Z$, so the number of vertices in $\Gamma(F(Y)) \cap B_{1}$ is $(6-\alpha) y+3-3 c_{F(Y)}-y-\left(2 y+1-c_{F(Y)}\right)-c_{Z}=(3-\alpha) y+2-2 c_{F(Y)}-c_{Z}$. If we take $X=\Gamma(Y)$, Equation 10 gives

$$
\begin{aligned}
(\alpha+1) y & \geq b_{0}(X) \geq b_{1}(X)-3+3 c_{F(X)} \\
& \geq(3-\alpha) y+2-2 c_{F(Y)}-c_{Z}-3+3 c_{F(X)} \\
& \geq(3-\alpha) y-1-2 c_{F(Y)}+2 c_{F(X)}
\end{aligned}
$$

where in the last inequality we use the fact that $c_{Z} \leq c_{F(X)}$. As $c_{F(Y)} \leq c_{F(X)}$, we have:

$$
\alpha \geq 1-\frac{1}{2 y} .
$$

We consider all the connected components of the graph induced by $B_{0} \cup A$ and we take a $q$-quasi-partition of the big components for some $q, q=O(k)$ such that 
all components are of the form $F(Y)$ for some $Y$ subset of $B_{0}$. Now we count the edges going to $B_{0}$ and $B_{1}$.

For one component $D$ of the quasi-partition with $y$ vertices of $B_{0}$, we find at least $(3+\alpha) y \geq 4 y-\frac{1}{2}$ edges toward $B_{0}$ and at most $2 y+\frac{7}{2}$ edges toward $B_{1}$ with one extremity in $D$.

Globally, if $m$ is the number of components and up to some small number of recounting (Quasi-partition arguments) we get $4|A|-\frac{m}{2}=3 b_{0}$ edges toward $B_{0}$ and $2 A+\frac{7 m}{2}=3 b_{1}$ edges toward $B_{1}$.

Hence $b_{0} \geq 2 b_{1}+O\left(\frac{1}{\sqrt{k}}\right)$.

\section{Conclusion}

In this paper we proposed constructions of valid $(p, \lambda, k)$-networks and gave lower bounds on their size. The design problem appears to be driven by two constraints: a local one in which small patterns are forbidden and another one which is related to some global expansion property of the network. This led us to define an expansion parameter of a graph: the $\alpha$-robustness. This parameter is a generalization of the usual edge-expansion. Using graphs of 2 -robustness equal to $\Theta(\log n)$ we constructed almost optimal simplified networks. Similarly when $k \leq \frac{n}{7}$, using graphs of large 1-robustness we proposed good simplified networks. Despite many answers for small values of $k\left(k \leq \frac{n}{7}\right)$, little is known when $k$ is larger.

To obtain our lower bounds, we presented a powerful technique: quasi-partitioning. We think that this technique can potentially have other applications to derive lower bounds for problems of the same kind.

Some interesting open questions remain

- For a fixed $\alpha$, find explicit constructions of nice $\alpha$-robust graphs, graphs having large $\alpha$-robustness.

- Is there a very explicit construction such as the "Zig-Zag product" of [29] and "lift" of [13] providing nice robust graphs? Does the Zig-Zag product preserve robustness?

- What is the maximum bisection-width of a 4-regular graph? In [24] the maximal bisection width of 4-regular graphs on $n$ vertices is shown to be at most $\frac{2 n}{5}$. This leads to an $\alpha$-robustness $<\frac{n}{2}$ when $\alpha>\frac{4}{5}$. Is it possible to obtain the same kinds of results for larger values of $\alpha$ ensuring a maximal robustness of size at most $\frac{n}{3}$, for example?

- What is the minimum number of switches or edges in an $n$-superselector? (See $[23,30]$ for some constructions.)

\section{Acknowledgments}

We are grateful to the anonymous referees for their valuable remarks which helped us to improve the presentation of our results. We especially thank Networks' Editorin-Chief Douglas Shier for his helpful comments on earlier versions of this paper. We also want to acknowledge Jean-Claude Bermond and Frédéric Havet for their interest in our work. 


\section{References}

[1] N. Alon, On the edge-expansion of graphs, Combin, Probability Comput 11 (1993), $1-10$.

[2] N. Alon and M. Capalbo, Smaller explicit superconcentrators, Proc 14th Ann Symp Discret Algorithms ACM-SIAM SODA (2003), 340-346.

[3] N. Alon, Z. Galil, and V.D. Milman, Better expanders and superconcentrators, J Algorithms 8 (1987), 337-347.

[4] N. Alon, P. Hamburger, and A.V. Kostochka, Regular honest graphs, isoperimetric numbers, and bisection of weighted graphs, Eur J Combin 20 (1999), 469-481.

[5] N. Alon and V.D. Milman, Eigenvalues, expanders and superconcentrators, Proc 25th Ann Symp Fondations Comput Sci (FOCS), Singer Island, Florida (1984), 320-322.

[6] L.A. Bassalygo, Asymptotically optimal switching circuits, Problemy Pederachi Informatsii 17 (1981), 81-88.

[7] B. Beauquier and E. Darrot, Arbitrary size Waksman networks, Première rencontres francophones sur les aspects algorithmiques de télécommunication (Algotel) (1999), 95-100.

[8] B. Beauquier and E. Darrot, Arbitrary size Waksman networks and their vulnerability, Parallel Process Lett 3-4 (2002), 287-296.

[9] J.C. Bermond, E. Darrot, and O. Delmas, Design of fault tolerant on-board networks in satellites, Networks 40 (2002), 202-207.

[10] J.C. Bermond, O. Delmas, F. Havet, M. Montassier, and S. Perennes, Réseaux de télécommunications minimaux embarqués tolérants aux pannes, Cinquième rencontres francophones sur les aspects algorithmiques de télécommunication (Algotel), (2003), 27-32.

[11] J.C. Bermond, F. Giroire, and S. Pérennes, Design of minimal fault tolerant on-board networks : Practical constructions, 14th Int Colloq Structural Informat Commun Complexity (SIROCCO), Lecture Notes in Comput Sci, Springer (2007), Number 4474, 261-273.

[12] J.C. Bermond, F. Havet, and C.D. Tóth, Fault tolerant on-board networks with priorities, Networks 47 (2006), 9-25.

[13] Y. Bilu and N. Linial, Lifts, discrepancy and nearly optimal spectral gaps, Combinatorica 26 (2006), 495-519.

[14] M. Blum, R. Karp, C. Papadimitriou, O. Vornberger, and M. Yannakakis, The complexity of testing whether a graph is a superconcentrator, Informat Process Lett 13 (1981), 164-167.

[15] B. Bollobás, The isoperimetric number of random regular graphs, Eur J Combin 9, 3 (1988), 241-244.

[16] M. Capalbo, O. Reingold, S. Vadhan, and A. Wigderson, Randomness conductors and constant-degree lossless expanders, 34th ACM Symp Theory Comput (STOCS) (2002), 659-668.

[17] F.R.K. Chung, On concentrators, superconcentrators, generalizers and nonblocking networks, Bell System Tech J 58 (1979), 1765-1777.

[18] F.R.K. Chung, Spectral Graph Theory, American Mathematical Society (1997).

[19] G. Davidoff, P. Sarnak, and A. Valette, Elementary number theory, group theory, and Ramanujan graphs, Cambridge University Press (2003).

[20] O. Delmas, F. Havet, M. Montassier, and S. Pérennes, Design of fault tolerant onboard networks, INRIA Res Report 5866, submitted (2006). 
[21] F. Giroire, Réseaux, algorithmique et analyse combinatoire de grands ensembles, Ph.D. Thesis, Université Paris VI (2006).

[22] C. Greenhill, J.H. Kim, and N.C. Wormald, Hamiltonian decompositions of random bipartite regular graphs, J Combinatorial Theory Ser B 90 (2004), 195-222.

[23] F. Havet, Repartitors, selectors and superselectors, J Interconnection Networks 7 (2006), 391-415.

[24] B. Monien and R. Preis, Upper bounds on the bisection width of 3-and 4-regular graphs, J Discr Algorithms 4 (2006), 475-498.

[25] M. Morgenstern, Existence and explicit constructions of $q+1$ regular Ramanujan graphs for every prime power $q$, J Combinatorial Theory Ser B 62 (1994), 44-62.

[26] M. Murty, Ramanujan graphs, J Ramanujan Math Soc 18 (2003), 33-52.

[27] H.Q. Ngo and D. Du, Notes on the complexity of switching networks, Advances in Switching Networks, Kluwer Academic Publishers, Dordrecht, The Netherlands (2001), 307-367.

[28] N. Pippenger, Superconcentrators, SIAM J Comput 6 (1977), 298-304.

[29] O. Reingold, S. Vadhan, and A. Wigderson, Entropy waves, the zig-zag graph product, and new constant-degree expanders and extractors, Ann Math 155 (2002), 157187.

[30] U. Schöning, Smaller superconcentrators of density 28, Informat Process Lett 98 (2006), 127-129.

[31] L.G. Valiant, On non-linear lower bounds in computational complexity, 7th ACM Symp Theory Comput (STOCS) (1975), 45-53. 